\title{
Human Bone Marrow-Derived Mesenchymal Stem Cell-Secreted Exosomes Overexpressing Microrna- 124-3p Inhibit DLBCL Progression By Downregulating NFATc1
}

\author{
Xiaolei Ding \\ The Second Hospital of Dalian Medical University \\ Lingzhi Xu \\ The Second Hospital of Dalian Medical University

\section{Xiuhua Sun} \\ The Second Hospital of Dalian Medical University

\section{Xiaoxuan Zhao} \\ Dalian Dermatosis Hospital
}

\section{Bing Gao}

Third Affiliated Hospital of Dalian Medical University

\section{Ye Cheng}

Third Affiliated Hospital of Dalian Medical University

\section{Dan Liu}

Second Affliated Hospital of Dalian Medical University: First Affiliated Hospital of Dalian Medical University

\section{Jian Zhao}

The Second Hospital of Dalian Medical University

\section{Xian Zhang}

The Second Hospital of Dalian Medical University

\section{Liye Xu}

The Second Hospital of Dalian Medical University

Jin-cheng Song ( $\square$ wwepqwq@163.com )

Second Affiliated Hospital of Dalian Medical University https://orcid.org/0000-0001-9263-4614

\section{Research}

Keywords: Human bone marrow mesenchymal stem cells, DLBCL, Exosomes, MicroRNA-124-3p, NFATc1

Posted Date: December 2nd, 2020 
DOI: https://doi.org/10.21203/rs.3.rs-117708/v1

License: (c) (1) This work is licensed under a Creative Commons Attribution 4.0 International License. Read Full License 


\section{Abstract}

Background: Exosomes play important roles in intercellular communication by delivering microRNAs (miRNAs) that mediate tumor initiation and development, including those in diffuse large B cell lymphoma (DLBCL). To date, however, limited studies on the inhibitory effect of exosomes derived from human bone marrow-derived mesenchymal stem cells (hBMSCs) on DLBCL progression have been reported. Therefore, this study aimed to investigate the role of hBMSC-secreted exosomes carrying microRNA-124-3p in the development of DLBCL.

Methods: Microarray-based expression analysis was adopted to identify differentially expressed genes and regulatory miRNAs, which revealed the candidate NFATc1. Next, the binding affinity between miR-124$3 p$ and NFATc1 was using luciferase activity assays. The mechanism underlying NFATc1 regulation was investigated using lentiviral transfections. Subsequently, DLBCL cells were cocultured with exosomes derived from hBMSCs transfected with a miR-124-3p mimic or control. Proliferation and apoptosis were measured in vitro. Finally, the effects of hBMSC-derived miR-124-3p on tumor growth were investigated in vivo.

Results: MiR-124-3p was downregulated while NFATc1 was upregulated in DLBCL cells. MiR-124-3p specifically targeted and negatively regulated the expression of NFATc1 in DLBCL cells, upregulated miR124-3p-inhibited DLBCL cell proliferation and promoted apoptosis. In addition, we found that hBMSCderived exosomes carrying miR-124-3p repressed DLBCL cell proliferation both in vitro and in vivo.

Conclusion: hBMSC-derived exosomal miR-124-3p represses the development of DLBCL through the downregulation of NFATc1.

\section{Background}

Diffuse large B cell lymphoma (DLBCL) accounts for 30-40\% of all lymphoma cases and has the characteristics of high malignancy, fast infiltrating growth, and poor prognosis [1]. The 5-year survival rate of late-stage DLBCL patients is less than $30 \%$. Although approximately half of all DLBCL patients can be cured by the current standard chemotherapeutic regimen of rituximab combined with cyclophosphamide, doxorubicin, vincristine, and prednisone (R-CHOP), more than $40 \%$ of patients still experience relapse/refractory issues [2,3]. Therefore, effective new treatment approaches that can be applied to improve patient survival or delay the recurrence of DLBCL are urgently needed.

Human mesenchymal stem cells (hMSCs) from adult bone marrow can be induced to differentiate into multiple mesenchymal tissues and then expanded and genetically modified in vitro, representing a potential therapeutic strategy for cancer patients(4). hBMSCs can migrate to tumor tissues, but the role of hBMSCs in tumorigenesis and cancer development is not well defined. Additional emerging evidence indicates that hBMSCs may be crucial for DLBCL development $[5,6]$. Therefore, better knowledge of these functions of hBMSCs in DLBCL could facilitate improved prognosis and suppress malignancy. Exosomes secreted by hBMSCs are often used experimentally as a potential therapeutic target for malignant tumors 
[7]. Exosomes are small, membrane-enclosed vesicles $(30-150 \mathrm{~nm})$ that are secreted by various cells and deliver intracellular contents, such as proteins, messenger RNAs (mRNAs), and microRNAs (miRNAs), from the originating cells to the recipient cells [8]. Exosomes, as a kind of natural carrier with targeting effects and drug delivery capability, have the characteristics of good histocompatibility, few side effects and high efficiency of internal delivery [9]. Therefore, exosomes have great potential in clinical applications.

As tumor suppressors or oncogenes, miRNAs are small noncoding RNA molecules that can regulate gene expression at the posttranscriptional level [10]. MiR-124-3p has been shown to act as a tumor suppressor in several tumors, including breast cancer [11], cervical cancer [12] and colorectal cancer [13]. According to a previous study, miR-124-3p is poorly expressed in DLBCL and is capable of inhibiting cellular proliferation by binding to NF-KB [14]. In the present study, we found that miR-124-3p negatively regulates nuclear factor of activated T cells $\mathrm{C} 1$ (NFATc1). As a potential oncogene, NFATc1 has been reported to be involved in the development of DLBCL. Moreover, it was found that NFATc1 can mediate the transcription of CMYC, which is associated with proliferation and apoptosis in DLBCL [15].

Based on the above information, we aimed to explore the therapeutic effect of miR-124-3p transferred by hBMSC-derived exosomes on DLBCL by regulating NFATc1 to provide novel potential therapeutic strategies.

\section{Materials And Methods}

\section{Microarray-based expression analysis}

We isolated peripheral blood exosomes from 7 healthy subjects and 7 DLBCL patients, and then we compared their gene expression profiles with a microRNA microarray. The limma software package was used to identify the differentially expressed genes (DEGs) with $|\log F C|>2$ and $p$ value $<0.05$ as the threshold [16], and the pheatmap package was employed to construct heatmaps of the DEGs. The DLBCL-related microarray profiles of GSE29493 and GSE40239 were retrieved from the Gene Expression Omnibus (GEO) database (https://www.ncbi.nlm.nih.gov/geo/). GSE29493 was used to confirm that micro-124 was differentially expressed in DLBCL samples and seven normal samples. GSE40239 was used to examine the prognostic value of micro-124 in DLBCL samples. The miRbase database (http://www.mirbase.org/), miRDB database (http://mirdb.org), and TargetScan database (http://www.targetscan.org) were employed to predict the miRNAs that might regulate NFATc1. The targeted binding site between NFATc1 and miR-124-3p was predicted through the microRNA database (http://www.microrna.org/). The prognostic genes were obtained from the GEO database (GSE10846) (thresholds: $p<0.05$ and HR $>1.5$ or $<0.5$ ) [17]. The differentially expressed genes were downloaded from the GEPIA website (http://gepia.cancer-pku.cn/) under the following parameters: $\log 2 \mathrm{FC}=1$, qvalue $=0.01$, differential methods $=$ ANOVA, and chromosomal distribution $=$ both [18]. Gene Ontology (GO) analyses were performed using the WebGestalt website (http://www.webgestalt.org/option.php). Kyoto Encyclopedia of Genes and Genomes (KEGG) pathways were analyzed using Metascape 
(http://metascape.org/gp/index.html\#/main/step1). A protein-protein interaction (PPI) network was constructed using the STRING database (http://www.string-db.org/) with an integrated score of 0.9 [19]. Data were imported into Cytoscape 3.7.2 software to analyze the network. The expression of NFATc1 in The Cancer Genome Atlas (TCGA) database was analyzed using the GEPIA database and Oncomine dataset (www.oncomine.org). Transcription factors were predicted through the hTFtarget website (http://bioinfo.life.hust.edu.cn/hTFtarget\#!/).

\section{Tissue sample collection}

Primary DLBCL tissues $(n=36)$ were obtained from patients who underwent surgical resection at the Lymphoma and Myeloma Diagnosis and Treatment Center, The Second Affiliated Hospital of Dalian Medical University between 2016 and 2019. The tissue samples were immediately washed with phosphate buffered saline (PBS) after surgery and then cut into small blocks and preserved in liquid nitrogen in a cryopreservation tube. All DLBCL cases were confirmed through a histopathological examination. None of the patients had received cancer treatment before surgery. Complete patient clinicopathological data were collected and followed up until May 31, 2020. Patients who died from nonDLBCL-related diseases were excluded from this study.

\section{Cell cultures}

Human SU-DHL-6 and SU-DHL-10 cells were a kind gift from Professors Jing Wei and Fang Wang of the Biology Laboratory of Sichuan University (Sichuan, China). The SU-DHL-6 cell line (Cobioer Biosciences Co., Ltd.; January 2018) and the SU-DHL-10 cell line (Chinese Academy of Sciences; July 2019) were authenticated using short tandem repeat authentication. The cells were cultured in Roswell Park Memorial Institute 1640 medium (HyClone, Logan, UT, USA). Cells were supplemented with $10 \%$ fetal bovine serum (FBS; HyClone) (SU-DHL-10) and 15\% FBS (SU-DHL-6) and maintained in a humidified atmosphere at $37^{\circ} \mathrm{C}$ with $5 \% \mathrm{CO}_{2}$. The medium was supplemented with 100 units $/ \mathrm{mL}$ penicillin and 100 $\mu \mathrm{g} / \mathrm{mL}$ streptomycin. BMSCs were extracted and purified from the bone marrow of healthy adults and then cultured in Dulbecco's modified Eagle's medium (DMEM)

\section{hBMSC isolation and culture[20]}

Under sterile conditions, $10 \mathrm{~mL}$ bone marrow was collected from the femoral shaft fracture end with a 20$\mathrm{mL}$ syringe (containing 2,000 IU heparin), after which the bone marrow cells were mixed quickly with heparin. Next, the samples were transferred into a $15 \mathrm{~mL}$ sterile centrifuge tube and centrifuged at $258 \times \mathrm{g}$ for $10 \mathrm{~min}$. The remaining cells were washed with DMEM 3 times and resuspended in $15 \mathrm{~mL}$ medium after the upper adipose tissues were removed. The same volume of Ficoll-Paque ${ }^{\text {TM }}$ PLUS lymphocyte isolate (density $1.077 \mathrm{~g} / \mathrm{ml}$ ) was added to the tube after which the suspension was centrifuged at $715 \times \mathrm{g}$ for $20 \mathrm{~min}$. Nucleated cells were noted to be located predominately in the boundary and upper liquids, while most of the erythrocytes had precipitated to the bottom. The nucleated cells were retrieved from the interface with a pipette and centrifuged at $178 \times \mathrm{g}$ for $8 \mathrm{~min}$. Next, $5 \mathrm{~mL}$ cell culture medium was added to resuspend the nuclear cells. The cell suspension $(10 \mu \mathrm{L})$ was mixed with $490 \mu \mathrm{L}$ PBS. Then, $10 \mu \mathrm{L}$ of the 
mixture was obtained for cell counting. Subsequently, the cells were incubated in a culture bottle $\left(1 \times 10^{5}\right.$ cells/bottle) with $5 \mathrm{~mL}$ DMEM-F12 culture medium at $37^{\circ} \mathrm{C}$ with $5 \% \mathrm{CO} 2$. After $24 \mathrm{~h}$, hBMSCs began to adhere to the wall, and half of the medium was discarded to remove nonadherent cells. After 4-7 days, when the adherent hBMSCs reached $80-90 \%$ confluence, the cells were subcultured again. Then, the hBMSCs were passaged every 2-3 days after purification and amplification. hBMSCs of the third to seventh passages were used for further experiments.

hBMSCs were subjected to osteogenic and adipogenic differentiation in OriCell ${ }^{\text {TM }}$ medium (Cyagen Biosciences Inc., Guangzhou, China) and then stained with alizarin red and oil red O. A flow cytometer (BD Biosciences Pharmingen, San Jose, CA, USA) was adopted to detect the hBMSC surface markers CD105, CD90, CD31, CD34, CD45, CD166, CD29, CD11b, HLA-DR, and CD73 (antibodies were purchased from BD Biosciences, USA).

\section{Transfection and lentiviral transduction}

DLBCL cells were inoculated into $60-\mathrm{mm}$ dishes $\left(1 \times 10^{6} \mathrm{cells} / \mathrm{dish}\right)$ or $100-\mathrm{mm}$ dishes $\left(2 \times 10^{6} \mathrm{cells} / \mathrm{well}\right) 1$ day prior to transfection. The cells were transfected with miR-124-3p/NC mimic, miR-124-3p/NC inhibitor, and sh-NFATc1/NC plasmids (GenePharma, Shanghai, China) in accordance with the instructions provided with Lipofectamine 2000 (Invitrogen, Carlsbad, CA, USA) when cell confluence was confirmed to have reached $60-80 \%$. The cells were analyzed and further examined $48 \mathrm{~h}$ after transfection. Lentivirus packaging was performed in 60-mm dishes containing PMD2G $(1 \mu \mathrm{g})$, psPAX2 $(3 \mu \mathrm{g})$ and pLenti6.3Luciferase/miR-124-3p (miR-NC/miR-124-3p) (4 $\mu \mathrm{g}$ ) plasmids. Twenty-four hours after transfection, the medium was replaced with fresh medium for another $24 \mathrm{~h}$ culture period. The changed medium was collected and mixed for target cell infection purposes. Lentivirus-transfected BMSCs were cultured overnight in 24-well plates at a density of $5 \times 10^{4}$ cells/well. Before transduction, medium containing 500 $\mu \mathrm{L}$ lentivirus supernatant, $500 \mu \mathrm{L}$ fresh culture medium, and $8 \mu \mathrm{g}$ polyacrylamide (Sigma, St. Louis, MO, USA) was used to assist the internalization of virus particles. The plates were then centrifuged at $2100 \mathrm{~g}$ at $37^{\circ} \mathrm{C}$ for $1 \mathrm{~h}$ and replaced with fresh medium.

\section{Dual-luciferase reporter assay}

The targeting relationship between miR-124-3p and NFATc1 was verified with a dual luciferase reporter gene assay. The artificially synthesized NFATC1 3'-untranslated region (3'-UTR) gene fragment was integrated into the psiCHECK-2 dual luciferase vector to construct psiCHECK-2-NFATc1-3'-UTR-WT (NFATc1-WT) and psiCHECK-2-NFATc1-3'-UTR-MUT (NFATc1-MUT). The wild-type (WT) and mutant (MUT) plasmids were cotransfected with the miR-124-3p mimic and mimic NC. After $24 \mathrm{~h}$ of transfection, the cells were collected and lysed. Luciferase activity was detected using a Dual Luciferase Reporter Kit (Promega, Madison, WI, USA). The experiments were repeated 3 times independently, and all investigations involving at least 6 wells were repeated.

\section{Exosome isolation}


Serum exosomes: Whole blood $(10 \mathrm{~mL})$ was collected from DLBCL patients and controls, and the serum was separated. Subsequently, exosomes were isolated from the serum using an ExoQuick Kit (EXOQ20A1, System Biosciences, Palo Alto, CA, USA) in strict accordance with the instructions provided by the manufacturer.

Exosomes of DLBCL cell culture: When BMSC confluence reached approximately $80 \%$, the medium was changed to $10 \%$ exosome-free FBS at $37^{\circ} \mathrm{C}$ in a $\mathrm{CO} 2$ incubator for $48 \mathrm{~h}$. The cell supernatant $(100 \mathrm{~mL})$ was collected and subsequently centrifuged at $300 \times \mathrm{g}$ for $10 \mathrm{~min}, 2000 \times \mathrm{g}$ for $10 \mathrm{~min}$, and $10000 \times \mathrm{g}$ for 30 min at $4{ }^{\circ} \mathrm{C}$. The supernatant was collected and filtered through a $0.22 \mu \mathrm{m}$ filter (Beyotime Institute of Biotechnology, Shanghai, China) and then ultracentrifuged at $100000 \times \mathrm{g}$ for 2 hours. The sediments containing the exosomes were resuspended in 50 to $100 \mu \mathrm{L}$ PBS and stored at $-80^{\circ} \mathrm{C}$.

\section{Observation of exosomes under an electron microscope and NTA}

Transmission electron microscopy was used to identify exosomes. The exosome suspension was fixed with $2 \%$ paraformaldehyde and then adsorbed onto carbon-coated copper grids. The copper grids containing exosomes were incubated with $1 \%$ glutaraldehyde for 5 minutes, ddH2O for 2 minutes, uranyl acetate $(\mathrm{pH}=7)$ for 5 minutes, and methylcellulose for 10 minutes. Finally, the samples were examined under an electron microscope (HITACHI, Tokyo, Japan) after drying at an accelerating voltage of $80 \mathrm{kV}$.

Exosomes were dissolved in $1 \mathrm{~mL}$ PBS and vortexed for $1 \mathrm{~min}$ to ensure uniform distribution. A Zeta View PMX110 nanoparticle tracking analyzer (Particle Metrix, Germany) was employed to measure the size distribution of exosomes.

\section{Internalization of exosomes by DLBCL cells}

DiO is used to track exosomes, as it can stably bind to the lipid region of the exosome lipid membrane. All experimental steps were performed following the instructions of the DIO-Membrane EVS Labeling and Purification Kit (Rengen Biosciences Co., Ltd., Liaoning, China). Briefly, $5 \mu \mathrm{L}$ DiO was incubated with $50 \mu \mathrm{l}$ reaction buffer solution. Next, the system was cultured with $50 \mu \mathrm{l}$ exosomes at a concentration of $5 \times 10^{9} / \mathrm{mL}$ for $30 \mathrm{~min}$ at room temperature and then centrifuged at $50 \times \mathrm{gg}$ for 90 seconds on a spin column. One hundred microliters of the exosome labeling preparation was carefully applied to the top of the column. The column was placed in a $1.5 \mathrm{ml}$ lightproof microcentrifuge tube and then centrifuged for 90 seconds at $50 \times \mathrm{g}$. The eluate was collected as purified labeled exosomes. The labeled exosomes were cocultured with DLBCL cells in serum-free medium. DLBCL cells were subsequently fixed with $4 \%$ paraformaldehyde, and nuclei were stained with 4,6-diamidino-2-phenylindole (DAPI). Finally, the capture of exosomes by DLBCL cells was observed under a fluorescence microscope (FV3000, Olympus, Tokyo, Japan).

\section{Coculture and inhibition of exosome secretion}


The exosomes extracted from miR-124-3p-transfected hBMSCs were inoculated with DLBCL cells in a 6well plate for $48 \mathrm{~h}$ (Fig. 7a). The cells were then divided into two groups: Exo-miR-NC+ DLBCL and ExomiR-124-3p + DLBCL. DLBCL cells were washed with PBS three times after incubation, and then subsequent experiments were carried out. miR-124-3p-transfected hBMSCs $\left(5 \times 10^{4}\right)$ and DLBCL cells $\left(1 \times 10^{5}\right)$ were cocultured in 6-well plates containing Transwell apical and basolateral chambers (Fig. 8a). The apical chamber was cultured with DMEM containing $10 \%$ serum, and the basolateral chamber was cultured in DMEM = containing 15\% serum. Cells were cocultured for $72 \mathrm{~h}$. After coculturing, hBMSCs and DLBCL cells were collected and used for subsequent experiments.

The specific inhibitors GW4869 (Sigma-Aldrich, St. Louis, MO, USA) and DMA (Santa Cruz, Paso Robles, CA, USA) were used to block exosome secretion. DMSO was used as a negative control. Then, $10 \mathrm{nM}$ GW4869 or 15 nM DMA was added to hBMSCs transfected with miR-124-3p at 48 hours prior to the end of coculture.

\section{Reverse transcription quantitative polymerase chain reaction (RT-qPCR)}

Total RNA was extracted using a TRIzol Kit (Invitrogen, Carlsbad, California, USA), and diethylpyrocarbonate (DEPC)-treated ultrapure water was used to dissolve RNA. RNA concentration and purity were measured on a Nanodrop 2000 ultraviolet spectrophotometer (Tiangen, Beijing, China) at wavelengths of $260 \mathrm{~nm}$ and $280 \mathrm{~nm}$. Next, the extracted RNA was reverse transcribed into complementary DNA (cDNA) in accordance with the instructions of the All-in-One miRNA RT-qPCR Kit (Takara, Shiga, Japan). Next, the reaction was performed according to the instructions of the kit (Fermentas Inc., Hanover, MD, USA). The primers were designed and synthesized by TaKaRa (Tokyo, Japan) (shown in Table 1). Real-time quantitative PCR (RT-qPCR) was conducted using a quantitative PCR instrument (Bio-Rad iQ5, Bio-Rad, Richmond, CA, USA). U6 was used as the internal reference for miR-124-3p, while glyceraldehyde-3-phosphate dehydrogenase (GAPDH) was used as the internal reference for NFATc1. The 2- $\Delta \Delta \mathrm{Ct}$ method was employed to determine relative gene expression. Each experiment was conducted 3 times.

\section{Western blot analysis}

Total protein was extracted and quantified using a bicinchoninic acid (BCA) kit (Thermo Fisher Scientific, Rockford, IL, USA). Protein samples $(30 \mu \mathrm{g})$ were used for sodium dodecyl sulfate (SDS)-polyacrylamide gel electrophoresis (PAGE) and subsequently transferred to a polyvinylidene fluoride (PVDF) membrane (Amersham plc, GE Healthcare, Chicago, Illinois, USA). Next, the proteins were blocked in bovine serum albumin (BSA) at room temperature for $1 \mathrm{~h}$ and incubated with primary antibodies against CD63 (1:1000, Abcam, UK), Hsp70 (1:1000, Abcam, UK), calnexin (1:1000, Abcam, UK), TSG 101 (1:1000, Abcam, UK),

cMYC (1:1000, Cell Signaling Technology Danvers, MA, USA), and GAPDH (1:5000, Abcam, UK) and a mouse antibody against NFATc1 (1:1000, Santa Cruz, CA, USA) at $4{ }^{\circ} \mathrm{C}$ overnight. The membranes were washed with TBST (TBS containing $0.1 \%$ Tween-20) three times followed by incubation with a horseradish peroxidase-conjugated secondary antibody for $1 \mathrm{~h}$ at room temperature. The samples were 
then washed with TBST 3 times and visualized by electrogenerated chemiluminescence detection, and the densitometric values were determined using a gel image analysis system (Bio-Rad Laboratories). Western blotting results were quantified using a Gel-Pro analyzer. The following formula was used to calculate the relative expression of the target protein: relative expression = gray value of the target protein band/ gray value of the internal reference band of the same sample.

\section{Apoptosis assay}

Apoptosis was assessed by annexin V-FITC and/or propidium iodide (PI) double standard staining analysis using flow cytometry. According to the manufacturer's instructions, cells $\left(1 \times 10^{6} \mathrm{cells} / \mathrm{mL}\right)$ were collected and washed three times with PBS. The cells were subsequently resuspended in $195 \mu \mathrm{L}$ binding buffer and gently mixed with $5 \mu \mathrm{L}$ annexin V-FITC and $10 \mu \mathrm{l} \mathrm{PI}$ at room temperature for $30 \mathrm{~min}$ in the dark. Cell apoptosis was analyzed using a BD FACSCanto II flow cytometer (BD Biosciences, San Jose, CA, USA) at $488 \mathrm{~nm}$. Data were analyzed with FlowJo software.

\section{Cell proliferation assay}

DLBCL cells were seeded at a density of $2 \times 10^{5}$ cells $/ \mathrm{mL}$ and incubated in 96-well plates for 24 and $48 \mathrm{~h}$. At the end of the incubation period, $10 \mu \mathrm{L}$ CCK-8 solution was added to each well and incubated at $37^{\circ} \mathrm{C}$ for an additional $2 \mathrm{~h}$ at $37^{\circ} \mathrm{C}$. The absorbance at $540 \mathrm{~nm}$ was recorded on a microplate reader (Bio-Rad Laboratories, Hercules, CA, USA).

\section{Pulldown assay}

The biotin-labeled double-stranded oligonucleotide probe targeting the CMYC promoter sequence was synthesized by PCR using biotin-labeled primers from Sangon Biotech Co. (sense, 5'-GCCCTATCAGAACAATGAAT- 3 ' and its complementary strand). Nuclear proteins $(400 \mu \mathrm{g})$ were mixed with the double-stranded biotinylated cMYC promoter probe $(4 \mu \mathrm{g})$ and streptavidin agarose beads $(50 \mathrm{ml})$ in 500 $\mathrm{ml}$ PBSI buffer containing $0.5 \mathrm{mM} \mathrm{PMSF}, 10 \mathrm{mM} \mathrm{NaF}, 25 \mathrm{mM}$ and $\beta$-glycerophosphate and rotated overnight at $4{ }^{\circ} \mathrm{C}$. The beads were centrifuged and washed with PBSI buffer two times, and then the beads were resuspended in loading buffer and boiled at $100^{\circ} \mathrm{C}$ for $10 \mathrm{~min}$. The supernatant was analyzed by western blotting.

\section{Tumor xenografts in nude mice}

A total of 12 male NOD/SCID mice (4-6 weeks old, weighing 18-22 g) were obtained from Huafukang Biotechnology Co., Ltd. (Beijing, China). One week after acclimation, SU-DHL-10 cells $\left(1 \times 10^{7}\right)$ were suspended in $100 \mu \mathrm{L}$ serum-free culture medium and subcutaneously injected into the upper flank region of NOD/SCID mice. Tumor volumes were measured every 2 days according to the formula $V=1 / 2$ * (short diameter) ${ }^{2} \times$ (longest diameter). After the tumor reached an approximate volume of $100 \mathrm{~mm}^{3}$, the mice were randomly allocated to three groups (MSC-derived miR-124-3p, MSC-derived miR-NC and PBS; four animals per group). hBMSC stable cell lines transfected with miR-124-3p or miR-NC were injected 
into NOD/SCID mice via the tail vein once every three days $\left(5 \times 10^{5}\right.$ cells/mouse). Subsequently, the tumor size was then measured. After seven injections, the mice were euthanized by means of anesthesia. Thereafter, tumors were collected and weighed. Finally, the tumor samples were frozen in liquid nitrogen or embedded in paraffin for immunohistochemistry analysis after imaging.

\section{Immunohistochemistry}

Immunohistochemical staining was performed using the streptavidin-peroxidase method. Formalin-fixed paraffin-embedded tissues were cut into $4 \mu \mathrm{m}$ thick sections that were dewaxed and dehydrated with gradient ethanol. Sections were pretreated with $10 \mathrm{mM}$ Tris-Na citrate $(\mathrm{pH} 6)$ for $20 \mathrm{~min}$ at $95^{\circ} \mathrm{C}$ and washed. Then, sections were incubated for 10 min in $3 \% \mathrm{H}_{2} \mathrm{O}_{2}$ in PBS to inhibit endogenous peroxidase. Next, the sections were subjected to antigen retrieval and blocking. The sections were subsequently incubated with primary antibodies against NFATc1 (1:50) and CMYC (1:200) diluted in 1\% BSA overnight at $4^{\circ} \mathrm{C}$ under agitation. After washing with PBS, the biotinylated secondary antibody-HRP conjugate (1:500) was incubated with normal goat serum (C-0005, Shanghai Haoran Bio Technology Co., Ltd., Shanghai, China) for 30 or 60 min at room temperature. The sections were developed with 3,3'diaminobenzidine and counterstained with hematoxylin (PT001, Shanghai Bogoo Biotechnology, Co., Ltd., Shanghai, China). After dehydration with gradient ethanol and sealing with neutral balsam, histological samples were quantified using light microscopy.

\section{Statistical analysis}

Statistical analyses were performed with SPSS 21.0 statistical software (SPSS, Inc., Chicago, IL, USA) and GraphPad Prism 6 software (GraphPad Software Inc., San Diego, CA, USA). Figures were generated with GraphPad Prism 6 software. Survival curves were drawn with the Kaplan-Meier method and analyzed by the log-rank test. Measurement data are presented as the mean \pm standard deviation (SD) of the experimental groups. Comparisons between two groups were performed with an independent samples $t$-test; comparisons between multiple groups were performed with one-way analysis of variance (ANOVA). $p<0.05$ indicated a statistically significant difference.

\section{Results}

\section{The expression of miR-124-3p is low in DLBCL tissues and cells}

A large number of differentially expressed microRNAs were obtained from the exosomes of DLBCL patients and healthy controls (Fig. 1b). Among these microRNAs, miR-124-3p drew our attention. The DLBCL-related microarray (GSE29493 and GSE2399) indicated that the expression of miR-124-3p was lower in DLBCL cells than in normal B cells (Fig. 1a and c). Furthermore, we observed that low miR-124-3p expression levels in DLBCL cells indicated a poorer prognosis and shorter overall survival (OS) than high miR-124-3p expression levels (GSE40239) (Fig. 1d). These results indicate that the expression level of miR-124-3p is relatively low in DLBCL. 
Overexpression of miR-124-3p inhibits proliferation and promotes apoptosis in DLBCL cells

To investigate the biological function of miR-124-3p, DLBCL cells were treated with a mimic and inhibitor of miR-124-3p. Compared with the mimic-negative control (NC) group, the apoptotic rate was elevated in the miR-124-3p mimic group ( $p$ 0.05) (Fig. 1e and $f$ ). When miR-124-3p was inhibited, cell proliferation was significantly increased (Fig. $1 \mathrm{~g}$ ). Thus, we demonstrated that upregulated miR-124-3p could inhibit DLBCL cell proliferation and facilitate apoptosis.

\section{MiR-124-3p may regulate the NFATc1 gene in DLBCL}

To further predict the target genes regulated by miR-124-3p, three miRNA-mRNA relation prediction databases (TargetScan, miRTarBase, and mirRDB) were used. A total of 1027 genes were identified as miR-124-3p-related genes (Fig. 2a). After intersection with the differentially expressed genes (GEPIA website) and prognostic genes (GSE10846), 237 genes were obtained (Fig. 2b). These genes are displayed in Fig. 2d. The genes were then subjected to GO, KEGG pathway, and PPI network analyses. GO functional enrichment analysis revealed that biological regulation, metabolic process, and response to stimulus were the main biological processes.

Concerning cellular components, the major components of these genes include membrane, nucleus, and membrane-enclosed lumen. These genes are related to various molecular function including protein binding, ion binding, and nucleic acid binding (Fig. 2c). PPI analysis using the STRING database and cytoHubba software showed that NFATc1 was one of the hub genes that exhibited the greatest number of interactions (Fig. 2e). Further KEGG enrichment analysis showed that these genes were mainly concentrated in the signaling pathways "blood vessel development", "regulation of cell adhesion" and "positive regulation of cell cycle" (Fig. 2f). Among these miR-124-3p-targeted genes, it was noted that NFATc1 is involved in multiple tumors, including DLBCL [21].

\section{MiR-124-3p targets and downregulates NFATc1}

The targeted binding site between NFATc1 and miR-124-3p was predicted through the microRNA database (http://www.microrna.org/) (Fig. 3a) and TargetScan (Fig. 3b). To verify that NFATc1 is the direct target gene of miR-124-3p, a dual luciferase reporter gene assay was performed. The results showed that compared to the NC group, the luciferase activity of' the NFATc1 wild-type (WT) 3'untranslated region (UTR) group was significantly inhibited by miR-124-3p $(p<0.05)$, while no difference was observed in the NFATc1 3'-UTR MUT group ( $p>0.05$ ) (Fig. 3c). To further verify the effect of miR-124$3 p$ on NFATc1, qRT-PCR (Fig. 3e) and western blot analysis (Fig. 3d and f) were then employed to determine the mRNA and protein expression of NFATc1. The expression of the NFATc1 gene was decreased when miR-124-3p was overexpressed, whereas this trend was reversed when miR-124-3p was inhibited. These findings indicate that NFATc1 is a target gene of miR-205 and that miR-205 negatively regulates NFATc1.

\section{Silencing NFATc1 inhibits proliferation and promotes apoptosis in DLBCL cells}


NFATC1 is highly expressed in DLBCL (TCGA database) (Fig. 4a). The Oncomine dataset showed that NFATc1 is differentially expressed (Fig. 4b) and associated with a poor prognosis (Fig. 4c). To verify the effect of NFATc1 on DLBCL cells, the NFATc1 gene was silenced. The silencing efficiency of sh-NFATc1 was evaluated by RT-PCR analysis (Fig. 4d). Silencing NFATc1 obviously suppressed cell proliferation (Fig. 4e) and promoted cell apoptosis (Fig. 4f). CMYC was one of the NFATc1-regulated genes predicted through the hTFtarget website, and the binding sites are displayed in Fig. 4g. There was a significant decline in C-MYC and NFATc1 expression in NFATc1-silenced cells (Fig. 4h). A pulldown assay was performed to analyze the binding of the NFATc1 protein to the CMYC promoter (Fig. 4h). NFATc1 knockdown suppressed proliferation and promoted apoptosis in DLBCL cells.

\section{Overexpression of NFATc1 rescues the biological function of miR-124-3p}

To investigate the relevance of miR-124-3p and NFATc1 in human DLBCL samples, we performed realtime PCR to determine the expression of miR-124-3p and NFATc1 in 36 DLBCL samples. There was a significant correlation of the miR-124-3p level with stage, IPI score, and extranodal invasion. The NFATc1 level was also correlated with stage and IPI score but not with other clinical features (Table 2). The Kaplan-Meier survival curve showed that increased miR-124-3p expression and low NFATc1 expression were related to good survival (Fig. $5 a$ and b). A negative correlation between miR-124-3p and NFATc1 was observed in the 36 DLBCL tissues ( $r=-0.707, p=0.001$, Fig. $5 c$ ). Furthermore, the miR-124-3p mimic was cotransfected with the NFATc1 overexpression plasmid. Exogenous overexpression of NFATc1 rescued the effects of miR-124-3p on DLBCL cells (Fig. $5 d$ and e). NFATc1 and cMYC protein expression was inhibited by miR-124-3p overexpression and reversed by NFATc1 cotransfection (Fig. $5 f$ ). These results indicate that the inhibitory role of miR-124-3p in DLBCL cells is mediated by the downregulation of NFATc1.

\section{Isolation and identification of hBMSCs}

After three to four passages, we observed a relatively large number of purified hBMSCs with a shuttle shape and swirling arrangement (Fig. 6b). Antibodies against CD105, CD90, CD31, CD34, CD 45, CD166, CD29, CD11b, HLA-DR, and CD73 were used to identify surface antigens by flow cytometry. Generally, CD29, CD90, CD73, CD105, and CD166 are considered markers of hBMSCs [22]. CD34 and CD45 are regarded as hematopoietic stem cell markers; CD31 is expressed ubiquitously within the vascular compartment and is located mainly at junctions between adjacent cells [23]. CD11b is expressed on the surface of many leukocytes, including monocytes, neutrophils, natural killer cells, granulocytes and macrophages, as well as on $8 \%$ of spleen cells and $44 \%$ of bone marrow cells. Functionally, CD $11 \mathrm{~b}$ regulates leukocyte adhesion and migration to mediate the inflammatory response [24]. HLA-DR is an MHC (major histocompatibility complex) class II cell surface receptor encoded by the human leukocyte antigen complex and is predominately expressed in lymphocytes and macrophages [25]. We found that CD29 (99.74\%), CD90 (99.81\%), CD105 (99.32\%), CD166 (96.33\%), and CD73 (99.73\%) were expressed, while CD34, CD31, CD45, CD11b, and HLA-DR were not. These results support that the cultured cells were hBMSCs (Fig. 6a). Next, the ability of hBMSCs to induce differentiation was assessed. We found that a 
large number of lipid droplets appeared in the cells after 2 weeks of adipogenic differentiation. Oil red 0 staining confirmed that the hBMSCs exhibited adipogenic differentiation (Fig. 6c). After 4 weeks of osteogenic differentiation, many red calcium nodules were observed the cell center using alizarin red staining (Fig. 6d).

\section{Exosomes are successfully extracted from hBMSCs}

Transmission electron microscopy (TEM) was used to identify BMSC-derived exosomes. The shape of exosomes was globular or oval, with a complete lipid membrane (Fig. 6e). The Zeta View nanoparticle tracking analyzer revealed that the exosome particles were predominately approximately $100 \mathrm{~nm}$ in size (Fig. 6f). Western blot analysis showed that the exosome surface marker proteins CD63, TSG 101, and Hsp70 were expressed in BMSC-derived exosomes, whereas calnexin was not (Fig. 6g). We found that the miR-124-3p level in miR-124-3p-transfected hBMSCs and the exosomes derived from miR-124-3p mimictreated hBMSCs was significantly higher than that in control cells (Fig. 6h). These results suggest that hBMSCs can effectively release exosomes containing miR-124-3p.

\section{hBMSCs deliver miR-124-3p to DLBCL cells by secreting exosomes}

Next, to observe whether exosomes derived from hBMSCs can be absorbed by DLBCL cells, we cocultured hBMSC-isolated exosomes labeled with DIO with DLBCL cells (Fig. 7a). As shown in Fig. 7b, slight green fluorescence, indicating exosomes absorbed by DLBCL cells, could be observed cells under a confocal fluorescence microscope. To determine whether miR-124-3p transferred from the exosomes of hBMSCs could modulate the biological function of DLBCL cells, proliferation and apoptosis experiments were conducted. The results showed that the apoptosis rates were increased (Fig. 7c) and the proliferation of DLBCL cells was inhibited (Fig. 7d) after hBMSC-derived exosomes containing miR-124-3p were added. Western blot analysis suggested that the protein expression of NFATc1 and CMYC was suppressed in DLBCL cells cocultured with hBMSC-derived exosomal miR-124-3p (Fig. 7e).

To further investigate the roles of hBMSC exosomes in DLBCL cells, the miR-124-3p-transfected hBMSCs were cocultured with DLBCL cells (Fig. 8a). Furthermore, we employed GW4869 and DMA, an exosome inhibitor, to reduce exosome secretion to determine the function of exosomes in this process. The results demonstrated that miR-124-3p-transfected hBMSCs inhibited proliferation (Fig. 8b) and induced apoptosis (Fig. 8c and d) in DLBCL cells by delivering miR-124-3p via the release of exosomes and that the biological function caused by hBMSC-derived exosomal miR-124-3p could be rescued by GW4869 or DMA. We also found that coculture with GW4869 and DMA resulted in a significant decrease in miR-124$3 p$ (Fig. 8e) and increase in NFATc1 (Fig. 8f). Thus, GW4869 and DMA could effectively inhibit the production of exosomes from hBMSCs and then reduce the transfer of miR-124-3p in hBMSCs to DLBCL cells, indicating that hBMSCs could affect the biological functions of DLBCL cells though exosomes.

\section{MiR-124-3p secreted from hBMSCs inhibits tumor growth in vivo}


To further evaluate the effect of hBMSC-derived miR-124-3p on DLBCL tumor growth in vivo, tumorbearing mice were injected with hBMSC-derived miR-124-3p, hBMSC-derived miR-NC or PBS via the tail vein. Tumor volume (Fig. 9a and b) and tumor weight (Fig. 9c) were measured, and the tumor tissues were subjected to immunohistochemical staining. The tumor volumes and tumor weights in the hBMSCderived miR-124-3p group were significantly lower than those in the hBMSC-derived miR-124-3p NC and PBS groups. These findings support the inhibitory effect of miR-124-3p on tumor growth in vivo. The immunohistochemical staining results showed that hBMSC-derived miR-124-3p significantly decreased the NFATc1 level in DLBCL tissues $(p<0.05)$ (Fig. 9d) compared with hBMSC-derived miR-NC and PBS.

Taken together, these results indicate that hBMSC-derived miR-124-3p suppresses tumor growth both in vitro and in vivo by downregulating NFATc1 (Fig. 10).

\section{Discussion}

DLBCL is one of the most aggressive lymphoid malignancies in humans. Although chemotherapy efficacy can be further improved by the application of rituximab, patients who experience relapse/refractory issues usually have a median survival duration of less than 2 years [3]. In recent years, MSC-derived exosomes transfected with miRNAs have been shown to play essential roles in tumorigenesis and can serve as mediators in human cancers. Several studies have reported that exosomes released from bone marrow mesenchymal stem cells (MSCs) can carry microRNAs (miRNAs), which are involved in cancer cell proliferation, differentiation, and apoptosis [26-28]. However, the effect of human BMSC (hBMSC)-derived exosomes on the occurrence and progression of DLBCL remains poorly understood. In our study, we explored the role of hBMSC-derived exosomes delivering miR-124-3p in DLBCL and found that miR-124-3p transferred by hBMSC-secreted exosomes could inhibit the proliferation of DLBCL cells by attenuating NFATC1 expression.

Our initial findings showed that miR-124-3p was downregulated while NFATc1 was upregulated in primary DLBCL tissues and DLBCL-derived cell lines. MiR-124-3p is a tumor suppressor microRNA with low expression in various hematological tumor types, including multiple myeloma (MM), acute myeloid leukemia (AML), acute lymphoblastic leukemia (ALL), chronic myeloid leukemia (CML), and B-cell lymphomas [29]. MiR-124-3p is frequently hypermethylated in non-Hodgkin's lymphoma (NHL), with a heterochromatic histone configuration. Hypermethylation results in the inactivation of miR-124-3p and the activation of downstream oncogenes, leading to tumorigenesis [30]. It has been reported that miR124-3p inhibits cell proliferation and promotes apoptosis by suppressing the expression of MYC and BCL2 by directly targeting NF-KB p65 in DLBCL cells [14]. In ovarian cancer, miR-124-3p secreted by ovarian surface epithelial cells can be transferred via exosomes to cancer-associated fibroblasts and inhibit the transition from normal fibroblasts to cancer-associated fibroblasts by targeting sphingosine kinase 1 (SPHK1) [31]. Our results also suggest that miR-124-3p can target and negatively regulate the expression of the transcription factor NFATC1. NFATc1 can directly upregulate the cMYC gene by binding to the CMYC promoter, and the upregulation of CMYC expression is closely related to the growth and survival of tumor cells [32,33]. In human acute myelogenous leukemia, high NFATc1 expression is 
associated with a poor prognosis [34]. However, NFATc1 overexpression is associated with good overall survival, and the ectopic expression of NFATc1 inhibits hepatic cancer proliferation [35]. Our results suggest that NFATc1 can promote the expression of c-MYC. Moreover, increased NFATc1 expression has been correlated with an advanced tumor stage, a high IPI score and a poor prognosis in DLBCL patients. Pham et al. found a novel epigenetic chromatin remodeling mechanism for NFATc1 in the pathophysiology of aggressive lymphoma B cells. NFATc1 regulates the c-myc oncogene in DLBCL cells through a chromatin remodeling mechanism that involves recruitment of the SWI/SNF ATPase Brg-1 chromatin remodeling complex [15]. Gong et al. concluded that miR-124-3p could target NFATc1 in the chondrogenesis process [36]. Until now, there has been no research focusing on miR-124-3p regulating NFATc1 in DLBCL. The bioinformatics analysis in the current study showed that NFATc1 was a target of miR-124-3p in DLBCL, which was further verified with a dual luciferase reporter gene assay. hBMSCs have self-renewal ability and multilineage differentiation potential and are able to differentiate into multiple cell types, including osteoblasts, adipocytes and chondroblasts [37].

The most commonly reported positive markers of hBMSCs are CD90, CD44, CD29, CD105, CD13, CD34, CD73, CD166, CD10, CD49e and CD59, while the most commonly reported negative markers are CD31, CD45, CD14, CD11b, CD34, CD19, CD56 and CD146. There are disagreements on the markers CD31, CD34, c-Kit (CD117) and STRO-1 [22]. We collected hBMSCs with positive CD29, CD90, CD105, CD166, and CD73 expression and negative CD34, CD31, CD45, CD11b, and HLA-DR expression. The ability to induce adipogenic differentiation and osteogenic differentiation further verified that the cultured cells were hBMSCs. BMSCs have been demonstrated to participate in the progression of multiple tumors through exosomes. Accumulating evidence indicates that exosomes secreted by cells play a crucial role in modulating intercellular communication and serve as important mediators in DLBCL $[38,39]$. A recent study suggested that exosomes derived from DLBCL cells expressed malignant molecules such as Bcl-2, c-Myc, Mcl-1, CD20, and CD19. DLBCL exosomes can be easily captured by lymphoma cells and DC cells. They mainly act as immunosuppressive mediators, as evidenced by the upregulation of PD-1 in T cells [40]. In addition, DLBCL exosomes promote cell proliferation, the migration of stromal cells and angiogenesis. It has also been demonstrated that exosomal miRNA-99a-5p and miRNA-125b-5p contribute to DLBCL chemoresistance [41]. Koch $\mathrm{R}$ et al [42] reported that DLBCL possesses a selforganized infrastructure comprising side population (SP) and non-SP cells. SP cells exhibit autonomous clonogenicity, and their clonogenic ability can be transported to non-SP cells by EXO-mediated Wnt signaling. Recent evidence has shown that miRNAs loaded into exosomes can be delivered to cancer cells and function as suppressors in vitro and in vivo. For instance, hBMSC-derived exosomal miR-205 retards prostate cancer progression by inhibiting RHPN2 [43]. MiR-155-5p secreted by melanoma cell exosomes can induce the proangiogenic switch of cancer-associated fibroblasts via the SOCS1/JAK2/STAT3 signaling pathway [44]. MiR-34a in hBMSC-derived exosomes inhibits glioblastoma cell proliferation, invasion, migration and tumorigenesis in vitro and in vivo and promotes chemosensitivity to temozolomide by silencing MYCN [20]. In the present study, we found that miR-124$3 p$ transferred by BMSC-derived exosomes could be delivered to DLBCL cells and inhibit tumor growth both in vivo and in vitro via the downregulation of NFATc1. 


\section{Conclusion}

In summary, the results of the study present show that miR-124-3p, when delivered by hBMSCs via exosomes, is able to inhibit proliferation and induce apoptosis in DLBCL cells by downregulating NFATc1. These findings likely highlight the potential of miR-124-3p as a novel molecular target for DLBCL treatment. However, the efficiency of miR-124-3p delivered from hBMSCs to DLBCL cells via exosomes needs to be validated and improved. Further research with more samples and more cell lines is required to substantiate our findings.

\section{Abbreviations}

DLBCL:Diffuse large B cell lymphoma; NFATc1: Nuclear factor of activated T cells c1; KEGG: Kyoto Encyclopedia of Genes and Genomes; TEM:Transmission electron microscope; MM: Multiple myeloma; AML: Acute myeloid leukaemia; ALL: Acute lymphoblastic leukaemia; CML: Chronic myeloid leukaemia; 3'UTR: 3'untranslated region; ANOVA: Analysis of variance; BCA: Bicinchoninic acid;; BMSCs: Bone marrow-derived mesenchymal stem cells; DAPI: 4,6-diamidino-2-phenylindole; DEGs: Differentially expressed genes; DEPC: Diethylpyrocarbonate; DMEM: Dulbecco's modified Eagle's medium; EDTA: Ethylenediaminetetraacetic acid; FBS: Fetal bovine serum; FITC: Fluorescein isothiocyanate; GAPDH: Glyceraldehyde-3-phosphate dehydrogenase; GEO: Gene Expression Omnibus; hBMSCs: Human bone marrow mesenchymal stem cells; hMSCs: Human mesenchymal stem cells; miRNAs: MicroRNAs; MSCs: Mesenchymal stem cells; MUT: Mutant; NC: Negative control; sh: short hairpin RNA; PBS: Phosphatebuffered saline; PBST: PBS containing 0.1\% Tween-20; PI: Propidium iodide; PVDF: Polyvinylidene fluorRT-qPCR: Reverse transcription quantitative polymerase chain reaction; SDS-PAGE: Sodium dodecyl sulfate polyacrylamide gelelectrophoresis; TCGA: The Cancer Genome Atlas; WT: Wild type

\section{Declarations}

\section{Acknowledgements}

We thank American Journal Experts (https://secure.aje.com/cn) for editing this manuscript. We would like to give our sincere appreciation to the colleagues for their valuable efforts and comments on this paper. Thanks to my wife for 4 years of support during my doctorate. This is a gift for our daughter.

\section{Author contributions}

JCS, LYX, XZ, XLD, LZX, and XHS designed the study. YC, DL, and BG collated the data, designed and developed the database, carried out data analyses. JZ and XXZ contributed to the drafting of the manuscript. All authors have contributed to the revision and approved the final submitted manuscript.

\section{Funding}


This work was supported by the Provincial Natural Science Foundation of Liaoning (No.2019-MS-096), Technological Innovation Foundation of Dalian (No. 2019J13SN87). Dalian Medical Science Research Project (No. 19Z12001), and Project of Western Medicine Bureau of Dalian Health Commission (No. 1911089).

\section{Ethics approval and consent to participate}

All participants enrolled in the study signed informed written consent documents, and the experimental procedures were in line with the Declaration of Helsinki. This study was approved by the Ethics Committee of China Medical University and The Second Affiliated Hospital of Dalian Medical University. All animal experiments were in strict accordance with the recommendations in the Guide for the Care and Use of Laboratory Animals of the National Institutes of Health.

\section{Availability of data and materials}

The datasets generated/analyzed during the current study are available.

\section{Consent for publication}

Not applicable.

\section{Competing interests}

The authors declare that they have no competing interests.

\section{References}

1. Castaneda-Ruiz P, Rada FVY, Serra-Jaramillo R, Paz-Cornejo E, Salas-Sanchez F. [Diffuse large B-cell lymphoma: a single disease?]. Rev Peru Med Exp Salud Publica. 2017;34(3):551-9.

2. Li S, Young KH, Medeiros LJ. Diffuse large B-cell lymphoma. Pathology. 2018;50(1):74-87.

3. Liu Y, Barta SK. Diffuse large B-cell lymphoma: 2019 update on diagnosis, risk stratification, and treatment. Am J Hematol. 2019;94(5):604-16.

4. Herrington CS, Poulsom R, Coates PJ. Recent Advances in Pathology: the 2020 Annual Review Issue of The Journal of Pathology. J Pathol. 2020;250(5):475-9.

5. Fastova EA, Magomedova AU, Petinati NA, Sats NV, Kapranov NM, Davydova YO, et al. Bone Marrow Multipotent Mesenchymal Stromal Cells in Patients with Diffuse Large B-Cell Lymphoma. Bull Exp Biol Med. 2019;167(1):150-3.

6. Zhong W, Zhu Z, Xu X, Zhang H, Xiong H, Li Q, et al. Human bone marrow-derived mesenchymal stem cells promote the growth and drug-resistance of diffuse large B-cell lymphoma by secreting IL- 6 and elevating IL-17A levels. J Exp Clin Cancer Res. 2019;38(1):73.

7. Zhu G, Pei L, Lin F, Yin H, Li X, He W, et al. Exosomes from human-bone-marrow-derived mesenchymal stem cells protect against renal ischemia/reperfusion injury via transferring miR-199a- 
3p. J Cell Physiol. 2019;234(12):23736-49.

8. Wei F, Li Z, Crawford R, Xiao Y, Zhou Y. Immunoregulatory role of exosomes derived from differentiating mesenchymal stromal cells on inflammation and osteogenesis. J Tissue Eng Regen Med. 2019;13(11):1978-91.

9. Xie C, Du LY, Guo F, Li X, Cheng B. Exosomes derived from microRNA-101-3p-overexpressing human bone marrow mesenchymal stem cells suppress oral cancer cell proliferation, invasion, and migration. Mol Cell Biochem. 2019;458(1-2):11-26.

10. Vishnoi A, Rani S. MiRNA Biogenesis and Regulation of Diseases: An Overview. Methods Mol Biol. 2017;1509:1-10.

11. Yan G, Li Y, Zhan L, Sun S, Yuan J, Wang T, et al. Decreased miR-124-3p promoted breast cancer proliferation and metastasis by targeting MGAT5. Am J Cancer Res. 2019;9(3):585-96.

12. Wang P, Zhang L, Zhang J, Xu G. MicroRNA-124-3p inhibits cell growth and metastasis in cervical cancer by targeting IGF2BP1. Exp Ther Med. 2018;15(2):1385-93.

13. Li Y, Dong W, Yang H, Xiao G. Propofol suppresses proliferation and metastasis of colorectal cancer cells by regulating miR-124-3p. 1/AKT3. Biotechnol Lett. 2020;42(3):493-504.

14. Jeong D, Kim J, Nam J, Sun H, Lee YH, Lee TJ, et al. MicroRNA-124 links p53 to the NF-kappaB pathway in B-cell lymphomas. Leukemia. 2015;29(9):1868-74.

15. Pham LV, Tamayo AT, Li C, Bueso-Ramos C, Ford RJ. An epigenetic chromatin remodeling role for NFATc1 in transcriptional regulation of growth and survival genes in diffuse large B-cell lymphomas. Blood. 2010;116(19):3899-906.

16. Ritchie ME, Phipson B, Wu D, Hu Y, Law CW, Shi W, et al. limma powers differential expression analyses for RNA-sequencing and microarray studies. Nucleic Acids Res. 2015;43(7):e47.

17. Song X, Du R, Gui H, Zhou M, Zhong W, Mao C, et al. Identification of potential hub genes related to the progression and prognosis of hepatocellular carcinoma through integrated bioinformatics analysis. Oncol Rep. 2020;43(1):133-46.

18. Zhou H, Yang Z, Yue J, Chen Y, Chen T, Mu T, et al. Identification of potential hub genes via bioinformatics analysis combined with experimental verification in colorectal cancer. Mol Carcinog. 2020;59(4):425-38.

19. Szklarczyk D, Morris JH, Cook H, Kuhn M, Wyder S, Simonovic M, et al. The STRING database in 2017: quality-controlled protein-protein association networks, made broadly accessible. Nucleic Acids Res. 2017;45(D1):D362-D8.

20. Wang B, Wu ZH, Lou PY, Chai C, Han SY, Ning JF, et al. Human bone marrow-derived mesenchymal stem cell-secreted exosomes overexpressing microRNA-34a ameliorate glioblastoma development via down-regulating MYCN. Cell Oncol (Dordr). 2019;42(6):783-99.

21. Song J, Zou D, Zhao X, Chen Y, Lv F, Wang S, et al. Bufalin inhibits human diffuse large B cell lymphoma tumorigenesis by inducing cell death through the Ca 2+/NFATC1/cMYC pathway. Carcinogenesis. 2020. 
22. Mildmay-White A, Khan W. Cell Surface Markers on Adipose-Derived Stem Cells: A Systematic Review. Curr Stem Cell Res Ther. 2017;12(6):484-92.

23. Dolmans MM, Cacciottola L, Amorim CA, Manavella D. Translational research aiming to improve survival of ovarian tissue transplants using adipose tissue-derived stem cells. Acta Obstet Gynecol Scand. 2019;98(5):665-71.

24. Lishko VK, Yakubenko VP, Ugarova TP, Podolnikova NP. Leukocyte integrin Mac-1 (CD11b/CD18, alphaMbeta2, CR3) acts as a functional receptor for platelet factor 4 . J Biol Chem. 2018;293(18):6869-82.

25. Tersigni C, Redman CW, Dragovic R, Tannetta D, Scambia G, Di Simone N, et al. HLA-DR is aberrantly expressed at feto-maternal interface in pre-eclampsia. J Reprod Immunol. 2018;129:48-52.

26. Xu YC, Lin YS, Zhang L, Lu Y, Sun YL, Fang ZG, et al. MicroRNAs of bone marrow mesenchymal stem cell-derived exosomes regulate acute myeloid leukemia cell proliferation and apoptosis. Chin Med $J$ (Engl). 2020.

27. Chen HL, Li JJ, Jiang F, Shi WJ, Chang GY. MicroRNA-4461 derived from bone marrow mesenchymal stem cell exosomes inhibits tumorigenesis by downregulating COPB2 expression in colorectal cancer. Biosci Biotechnol Biochem. 2020;84(2):338-46.

28. Qin F, Tang H, Zhang Y, Zhang Z, Huang P, Zhu J. Bone marrow-derived mesenchymal stem cellderived exosomal microRNA-208a promotes osteosarcoma cell proliferation, migration, and invasion. J Cell Physiol. 2020;235(5):4734-45.

29. Yang T, Jin X, Lan J, Wang W. Long non-coding RNA SNHG16 has Tumor suppressing effect in acute lymphoblastic leukemia by inverse interaction on hsa-miR-124-3p. IUBMB Life. 2019;71(1):134-42.

30. Wong KY, So CC, Loong F, Chung LP, Lam WW, Liang R, et al. Epigenetic inactivation of the miR-124-1 in haematological malignancies. PLoS One. 2011;6(4):e19027.

31. Zhang Y, Cai H, Chen S, Sun D, Zhang D, He Y. Exosomal transfer of miR-124 inhibits normal fibroblasts to cancer-associated fibroblasts transition by targeting sphingosine kinase 1 in ovarian cancer. J Cell Biochem. 2019;120(8):13187-201.

32. Xu W, Gu J, Ren Q, Shi Y, Xia Q, Wang J, et al. NFATC1 promotes cell growth and tumorigenesis in ovarian cancer up-regulating c-Myc through ERK1/2/p38 MAPK signal pathway. Tumour Biol. 2016;37(4):4493-500.

33. Buchholz M, Schatz A, Wagner M, Michl P, Linhart T, Adler G, et al. Overexpression of c-myc in pancreatic cancer caused by ectopic activation of NFATc1 and the $\mathrm{Ca} 2+$ /calcineurin signaling pathway. EMBO J. 2006;25(15):3714-24.

34. Solovey M, Wang Y, Michel C, Metzeler KH, Herold T, Gothert JR, et al. Nuclear factor of activated Tcells, NFATC1, governs FLT3(ITD)-driven hematopoietic stem cell transformation and a poor prognosis in AML. J Hematol Oncol. 2019;12(1):72.

35. Xu S, Shu P, Zou S, Shen X, Qu Y, Zhang Y, et al. NFATc1 is a tumor suppressor in hepatocellular carcinoma and induces tumor cell apoptosis by activating the FasL-mediated extrinsic signaling pathway. Cancer Med. 2018;7(9):4701-17. 
36. Gong M, Liang T, Jin S, Dai X, Zhou Z, Gao M, et al. Methylation-mediated silencing of miR-124 facilitates chondrogenesis by targeting NFATc1 under hypoxic conditions. Am J Transl Res. 2017;9(9):4111-24.

37. Graneli C, Thorfve A, Ruetschi U, Brisby H, Thomsen P, Lindahl A, et al. Novel markers of osteogenic and adipogenic differentiation of human bone marrow stromal cells identified using a quantitative proteomics approach. Stem Cell Res. 2014;12(1):153-65.

38. Ofori K, Bhagat G, Rai AJ. Exosomes and Extracellular Vesicles as Liquid Biopsy Biomarkers in Diffuse Large B-cell Lymphoma (DLBCL): Current State of the Art and Unmet Clinical Needs. Br J Clin Pharmacol. 2020.

39. Zare N, Haghjooy Javanmard SH, Mehrzad V, Eskandari N, Andalib AR. Effect of Plasma-Derived Exosomes of Refractory/Relapsed or Responsive Patients with Diffuse Large B-Cell Lymphoma on Natural Killer Cells Functions. Cell J. 2020;22(1):40-54.

40. Chen Z, You L, Wang L, Huang X, Liu H, Wei JY, et al. Dual effect of DLBCL-derived EXOs in lymphoma to improve DC vaccine efficacy in vitro while favor tumorgenesis in vivo. J Exp Clin Cancer Res. 2018;37(1):190.

41. Feng $Y$, Zhong M, Zeng S, Wang L, Liu P, Xiao X, et al. Exosome-derived miRNAs as predictive biomarkers for diffuse large B-cell lymphoma chemotherapy resistance. Epigenomics. 2019;11(1):35-51.

42. Koch R, Demant M, Aung T, Diering N, Cicholas A, Chapuy B, et al. Populational equilibrium through exosome-mediated Wnt signaling in tumor progression of diffuse large B-cell lymphoma. Blood. 2014;123(14):2189-98.

43. Jiang S, Mo C, Guo S, Zhuang J, Huang B, Mao X. Human bone marrow mesenchymal stem cellsderived microRNA-205-containing exosomes impede the progression of prostate cancer through suppression of RHPN2. J Exp Clin Cancer Res. 2019;38(1):495.

44. Zhou X, Yan T, Huang C, Xu Z, Wang L, Jiang E, et al. Melanoma cell-secreted exosomal miR-155-5p induce proangiogenic switch of cancer-associated fibroblasts via SOCS1/JAK2/STAT3 signaling pathway. J Exp Clin Cancer Res. 2018;37(1):242.

\section{Figures}


A

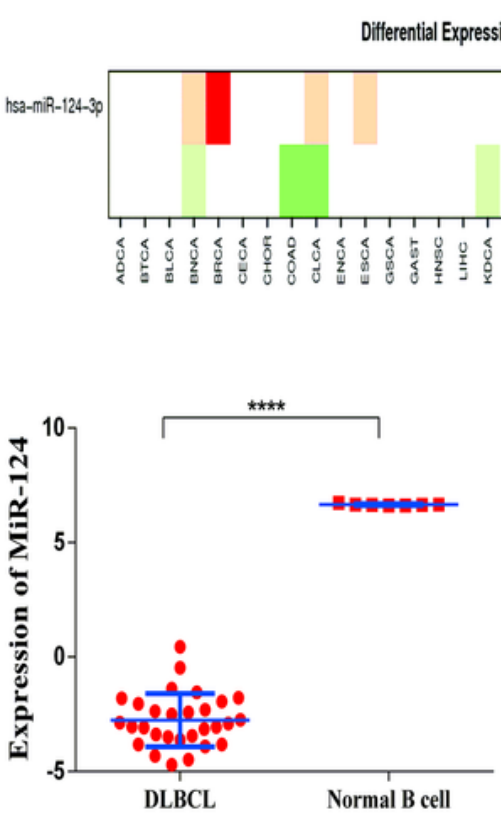

$\mathbf{E}$

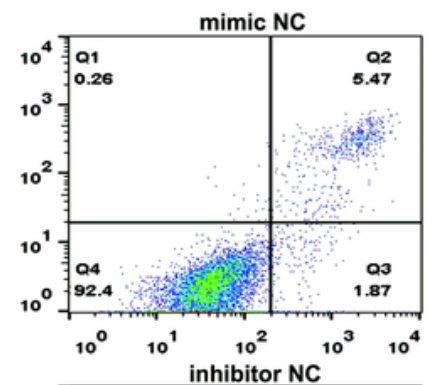

PI

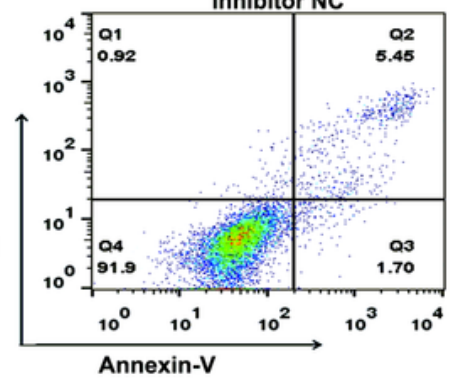

$\mathbf{F}$

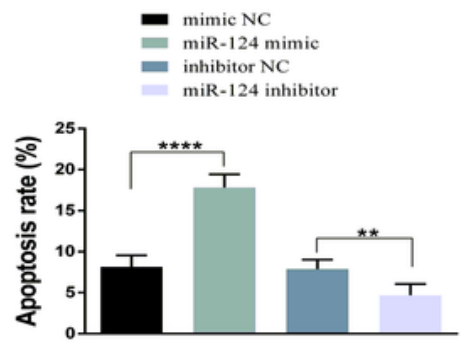

D
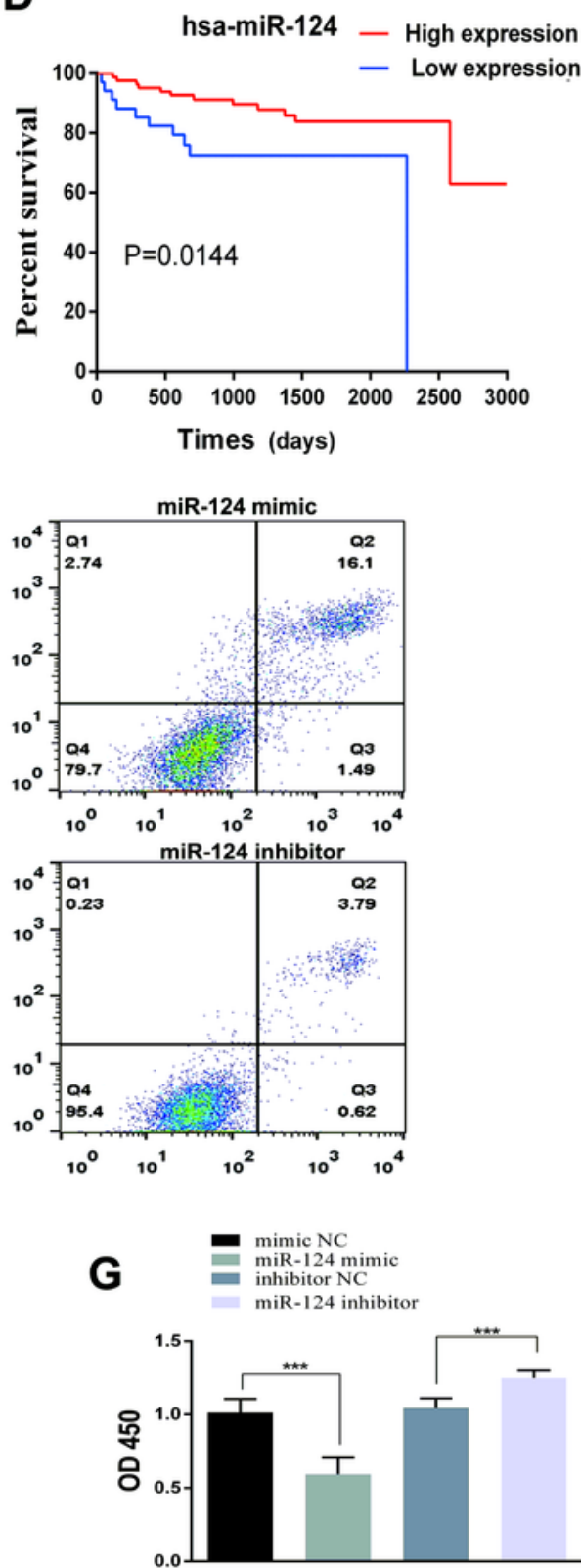

B

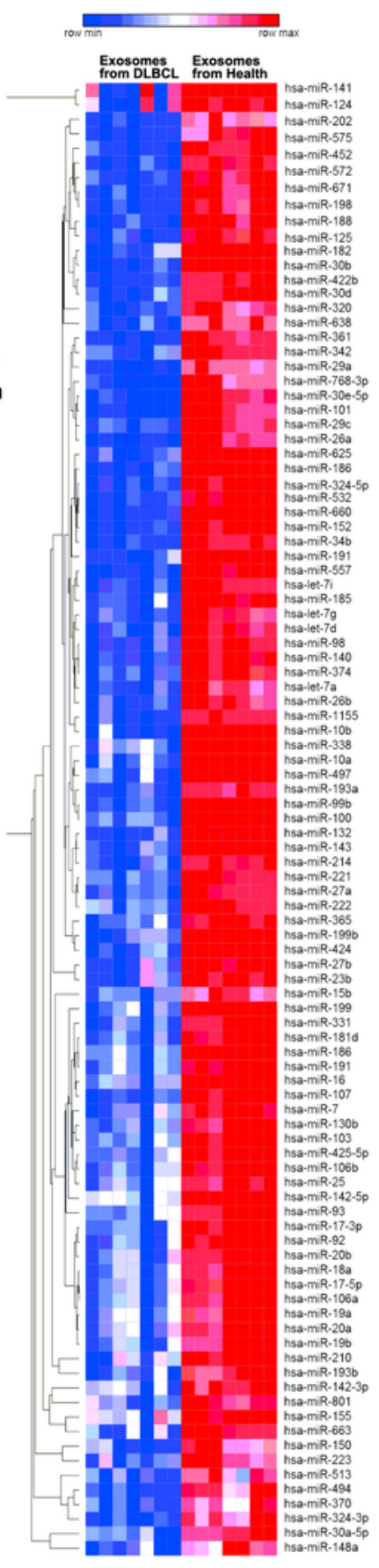

\section{Figure 1}

Low miR-124-3p expression in DLBCL tissues. a Differential expression profile of miR-124-3p in different cancers and normal tissues (GSE2399). b Differentially expressed microRNAs in the exosomes of DLBCL patients and healthy controls. The results indicate that miR-124-3p is expressed at low levels in the serum exosomes of DLBCL patients. c The expression of miR-124-3p is lower in DLBCL cells than in normal B cells (GSE29493). d Low miR-124-3p expression levels in DLBCL indicate a poorer prognosis and shorter 
overall survival (OS) than high miR-124-3p expression levels (GSE40239). e Overexpression of miR-124$3 p$ promotes apoptosis in DLBCL cells. f Quantification of figure e. $g$ Upregulated miR-124-3p inhibits DLBCL cell proliferation.

A

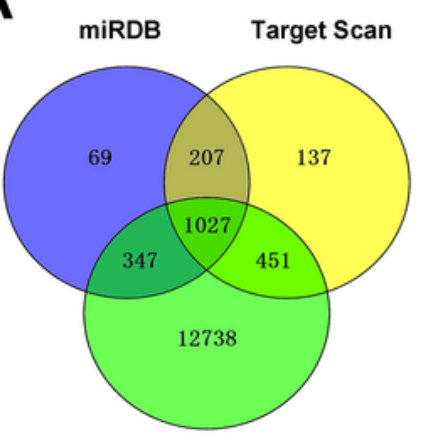

miRbase
B

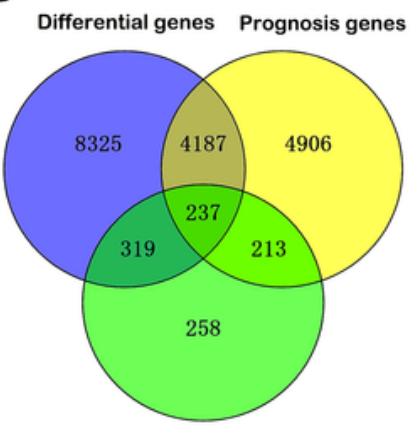

miR-124 target genes
C

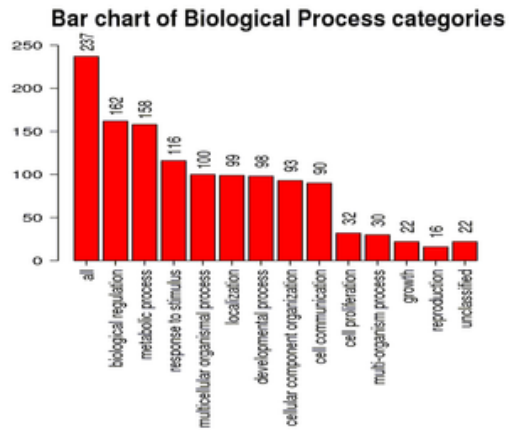

Bar chart of Cellular Component categories

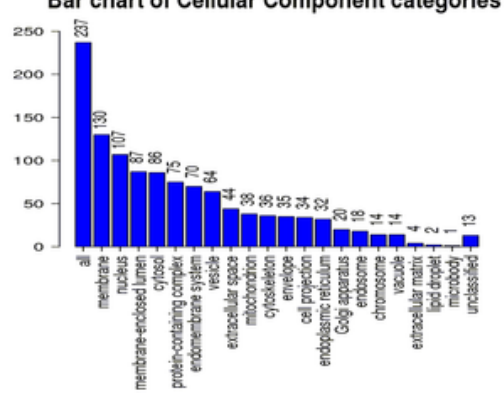

Bar chart of Molecular Function categories

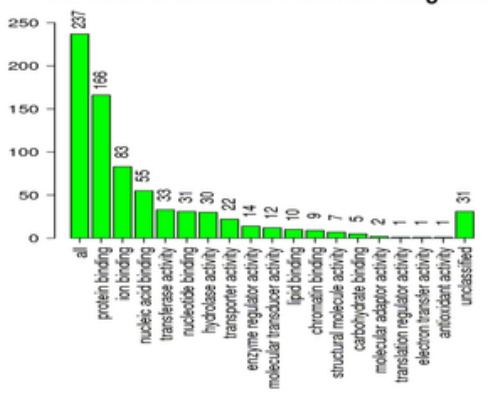

$\mathbf{E}$

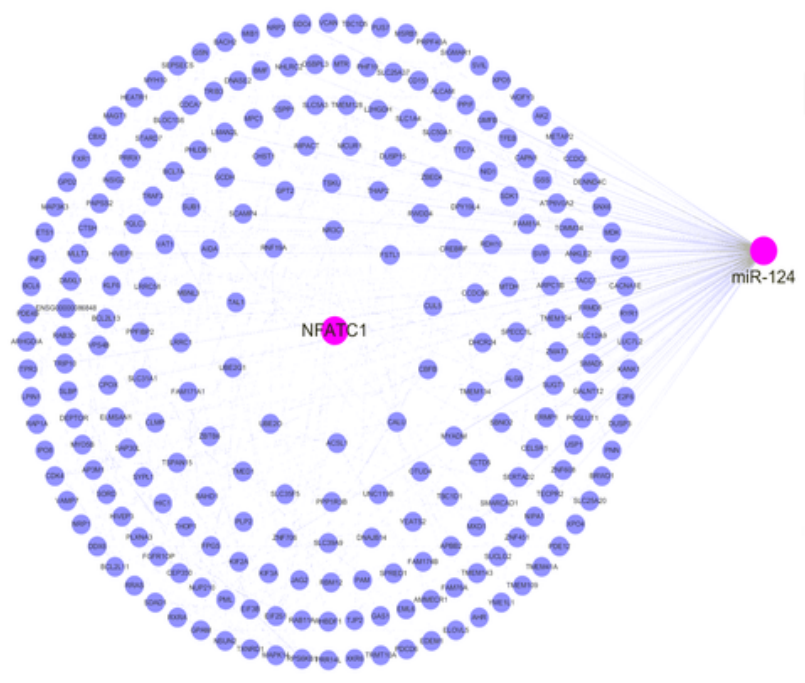

$\mathbf{F}$

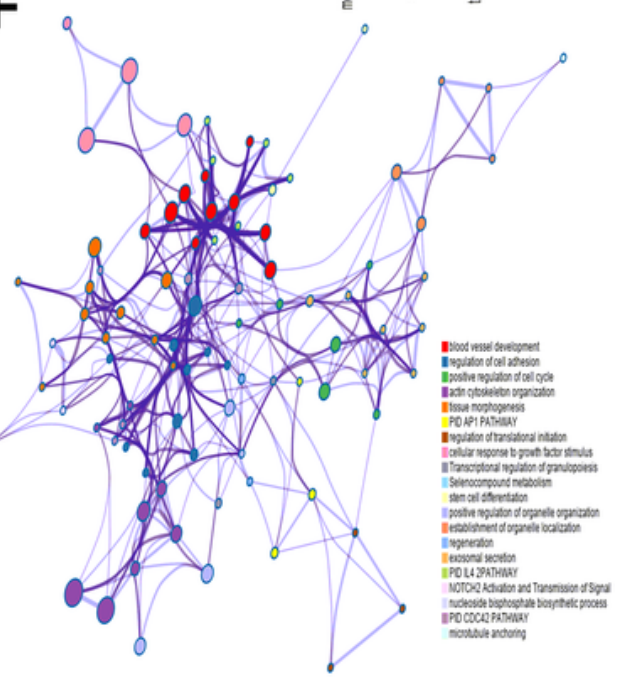

Figure 2

Bioinformatics predicted NFATc1 as the target of miR-124-3p. a A total of 1027 genes were identified as miR-124-3p-related genes analyzed by three miRNA-mRNA relation prediction databases (TargetScan, 
miRTarBase, and mirRDB). b The 1027 miR-124-3p-related genes were intersected with the differentially expressed genes (GEPIA website) and prognostic genes (GSE10846) in DLBCL. We obtained 237 miR124-3p-targeted genes in DLBCL. c GO functional enrichment analysis of the 237 miR-124-3p-targeted genes. The results revealed that biological regulation, metabolic process, and response to stimulus were the main biological processes. These genes are related to various cellular components, including membrane, nucleus, and membrane-enclosed lumen. Concerning molecular function, the major activities of these genes include protein binding, ion binding, and nucleic acid binding. $d$ The 237 miR-124-3prelated genes are displayed. e Protein-protein interaction (PPI) network analysis showed that NFATc1 was one of the hub genes that exhibited the greatest number of interactions analyzed using the STRING database and cytoHubba software. f Kyoto Encyclopedia of Genes and Genomes (KEGG) pathway analysis suggested that the $237 \mathrm{miR}-124-3 p$-related genes were mainly concentrated in the signaling pathways "blood vessel development", "regulation of cell adhesion" and "positive regulation of cell cycle". 
A

\section{C hsa-miR-124/NFATC1 Alignment}

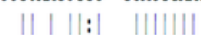
386:5' uUCAAU-ACUGGAGGUGCCUJa 3' NEATCI

C

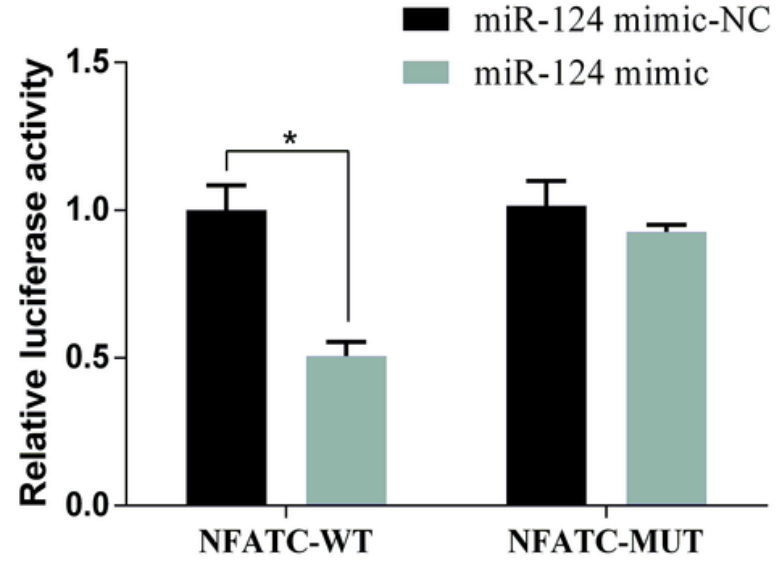

E

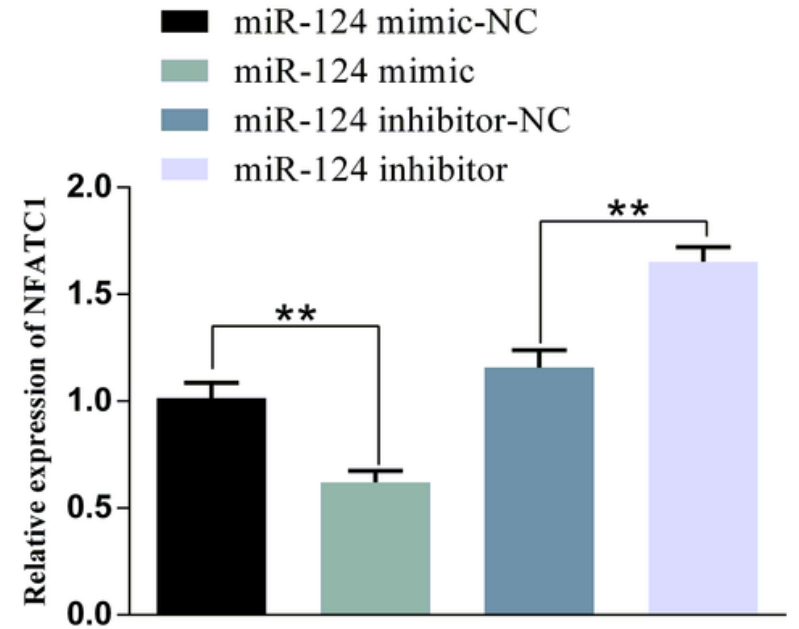

B
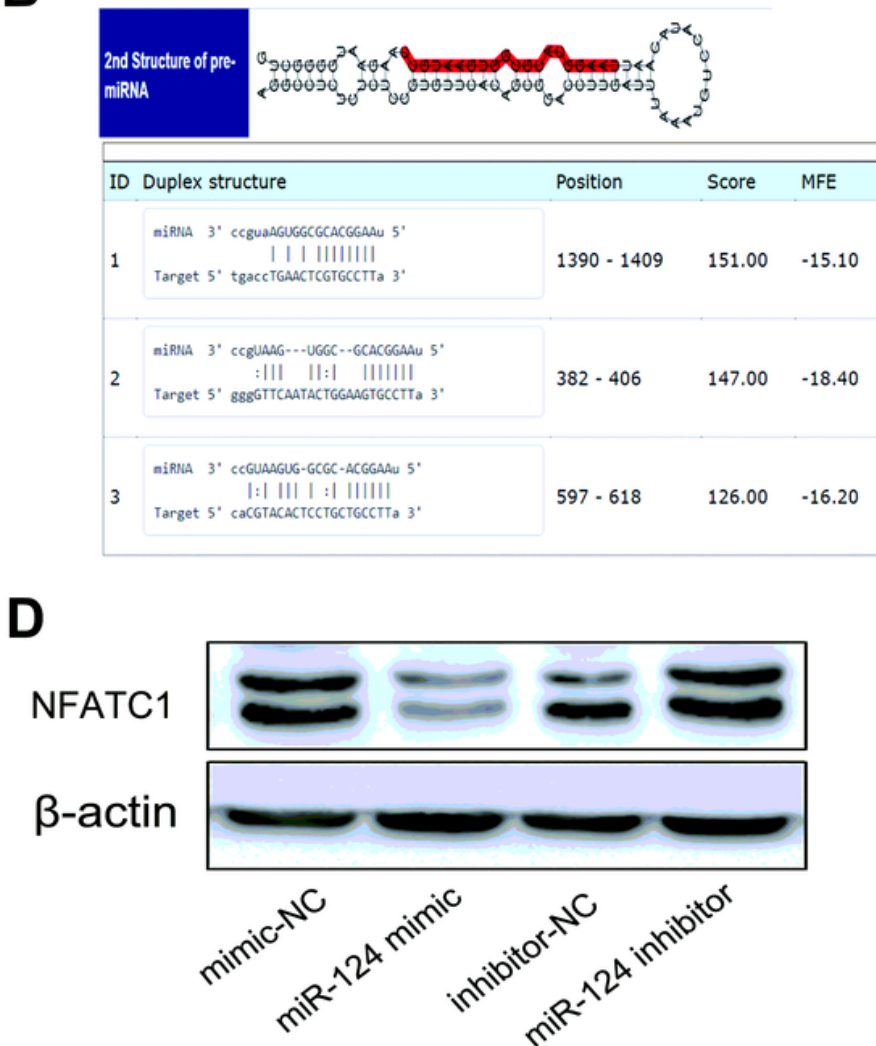

$\mathbf{F}$

- miR-124 mimic-NC

- miR-124 mimic

- miR-124 inhibitor-NC

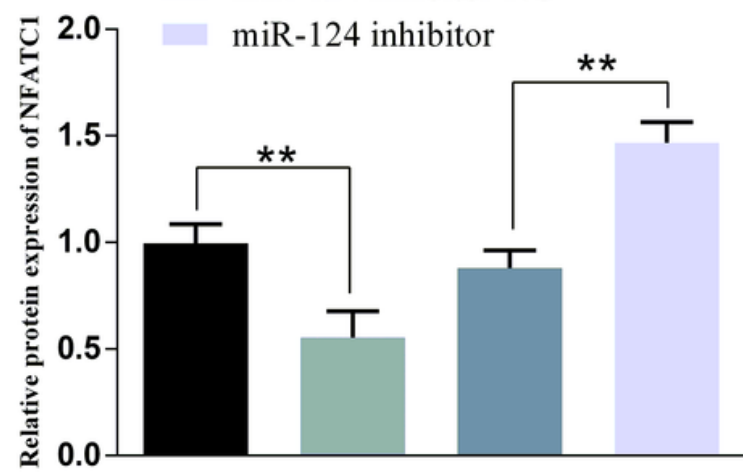

\section{Figure 3}

MiR-124-3p targets and negatively regulates NFATc1. a-b The targeted binding site between NFATc1 and miR-124-3p was predicted through the microRNA database and TargetScan database. c The target relationship between miR-124-3p and NFATc1 was confirmed by a dual-luciferase reporter gene assay. d-e The expression of NFATc1 was detected by RT-PCR (e) and western blot (d). f Quantification of figure d. The expression of the NFATc1 gene was decreased when miR-124-3p was overexpressed, whereas this trend was reversed when miR-124-3p was inhibited. The data are expressed as the mean \pm SD; comparisons between two groups were analyzed using unpaired t-tests, and the experiment was repeated three times independently ( $\left.{ }^{\mathrm{P}}<0.05, * * \mathrm{P}<0.01\right)$. 

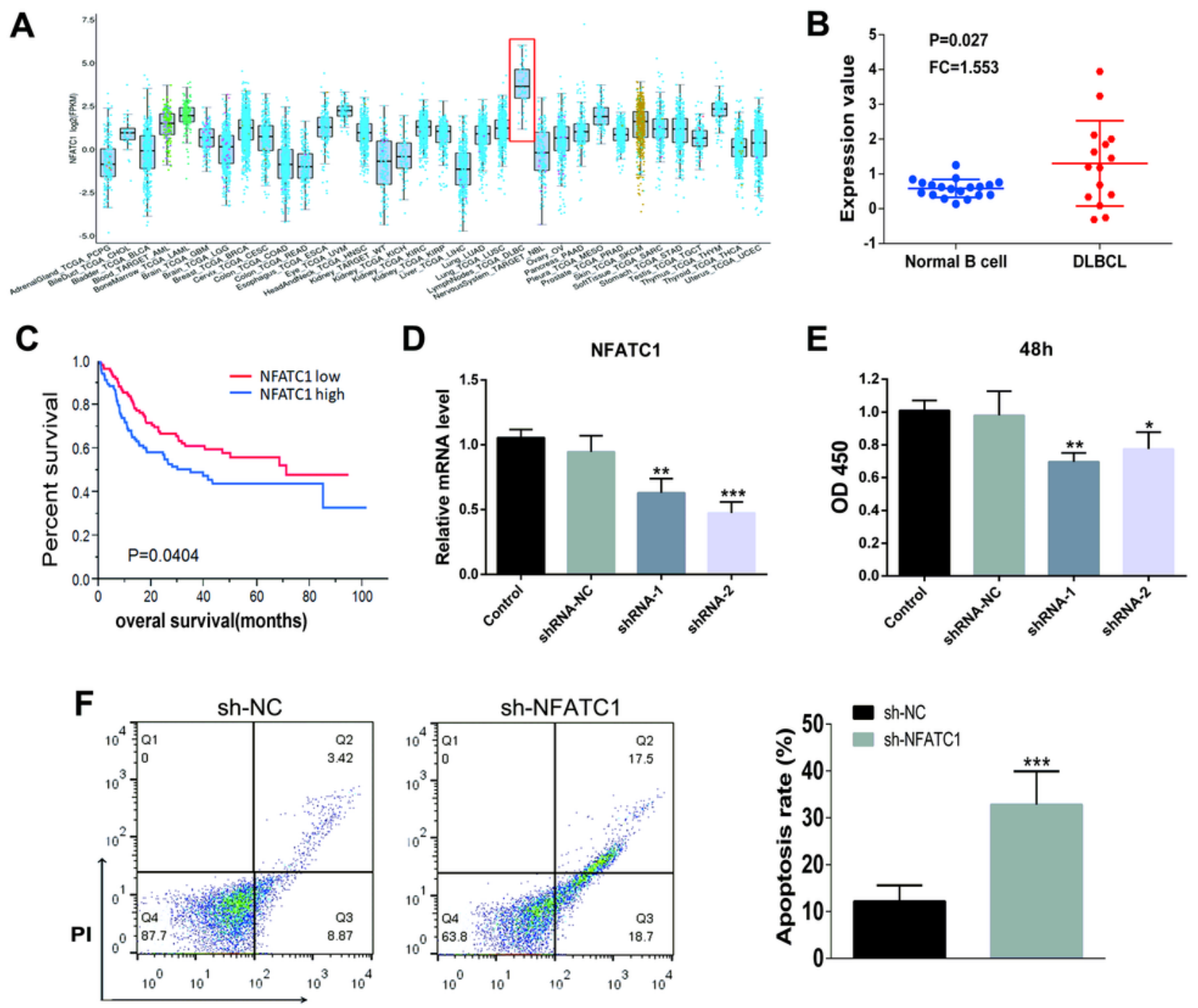

Annexin-V

G

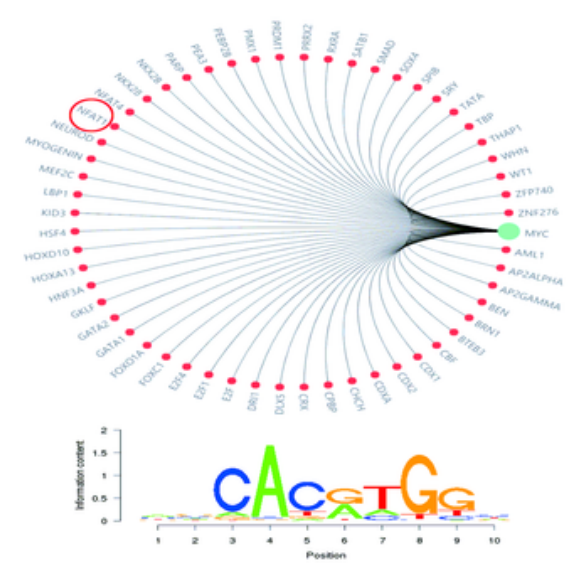

H
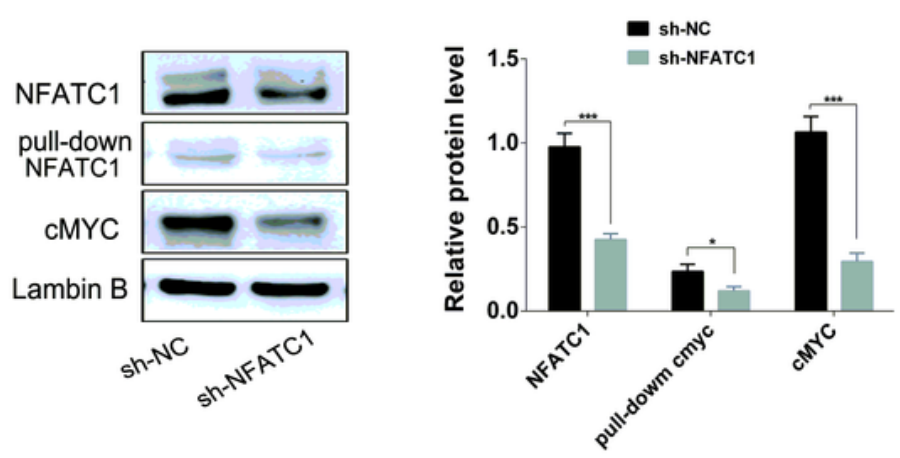

\section{Figure 4}

Silencing NFATc1 inhibits proliferation and promotes apoptosis in DLBCL cells. a The expression profile of NFATc1 in different cancers (TCGA database). b NFATc1 is highly expressed in DLBCL (Oncomine dataset). $c$ Patients with high NFATc1 expression have a poor prognosis. $d$ The silencing efficiency of shNFATc1 was evaluated by RT-PCR. e Proliferation was suppressed when NFATc1 was silenced. $f$ Silencing NFATc1 promoted cell apoptosis. g cMYC was one of the NFATC1-regulated genes predicted through the 
hTFtarget website, and the binding sites are displayed. h NFATc1 and CMYC protein expression in cells after silencing NFATc1 determined by western blot analysis. A pulldown assay was performed to analyze the binding of the NFATc1 protein to the CMYC promoter. Data are presented as the mean \pm SD of three independent experiments $\left({ }^{*} \mathrm{P}<0.05,{ }^{*} \mathrm{P}<0.01,{ }^{*} * \mathrm{P}<0.001\right)$.
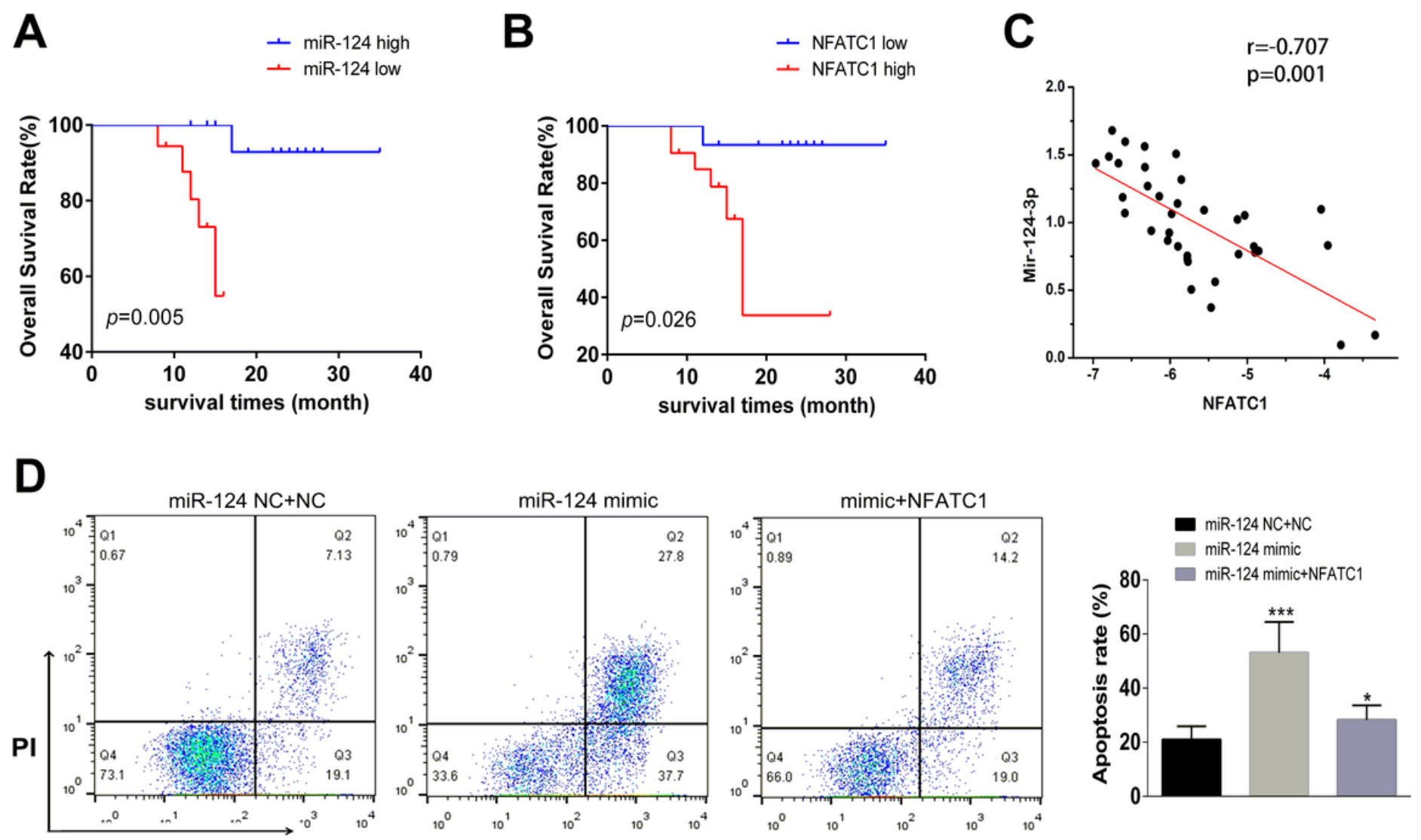

Annexin-V
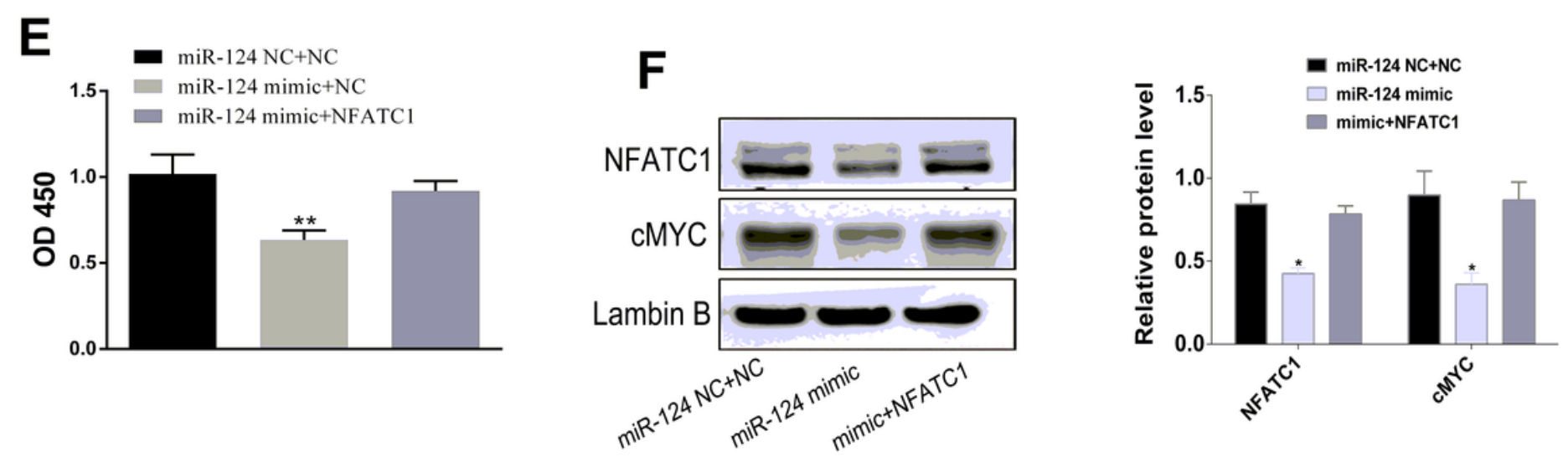

Figure 5

The biological function of miR-124-3p was reversed by NFATc1. a Our data suggest that high miR-124-3p expression is associated with a good prognosis. b High NFATc1 expression indicates a poor prognosis in our own data. c A negative correlation between miR-124-3p and NFATc1 was observed in 36 DLBCL tissues. $d$ The increased apoptosis observed by miR-124-3p overexpression was reversed by NFATc1 cotransfection. e The inhibitory effect of miR-124-3p upregulation on DLBCL cell proliferation was 
significantly reversed by NFATc1 overexpression. $\mathrm{f} \mathrm{NFATc1}$ and cMYC protein expression was inhibited by miR-124-3p overexpression and reversed by NFATc1 cotransfection. Data are presented as the mean \pm SD of three independent experiments $\left({ }^{*} \mathrm{P}<0.05,{ }^{* *} \mathrm{P}<0.01\right.$, $\left.{ }^{* *} \mathrm{P}<0.001\right)$.
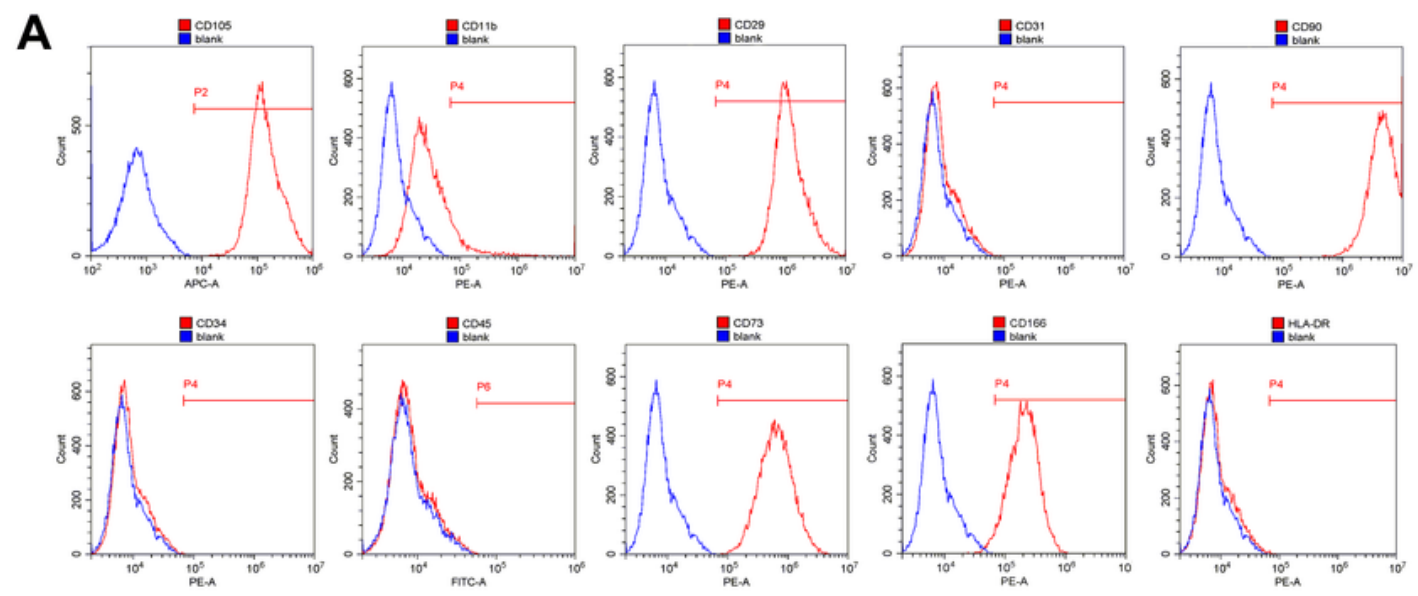

B

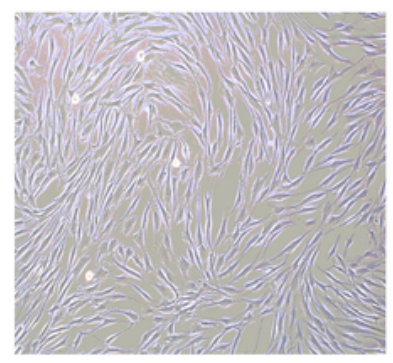

C

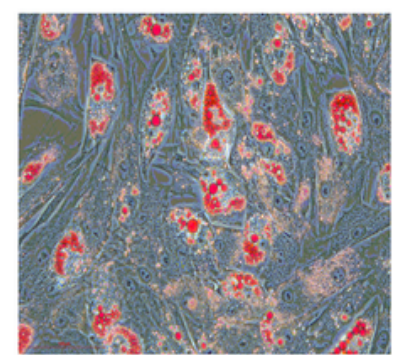

D

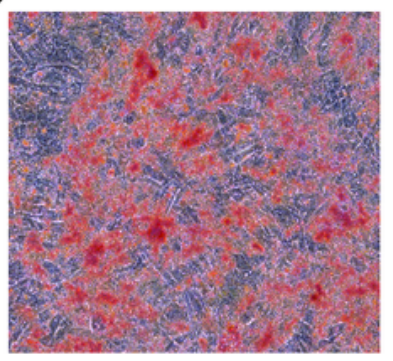

$\mathbf{F}$
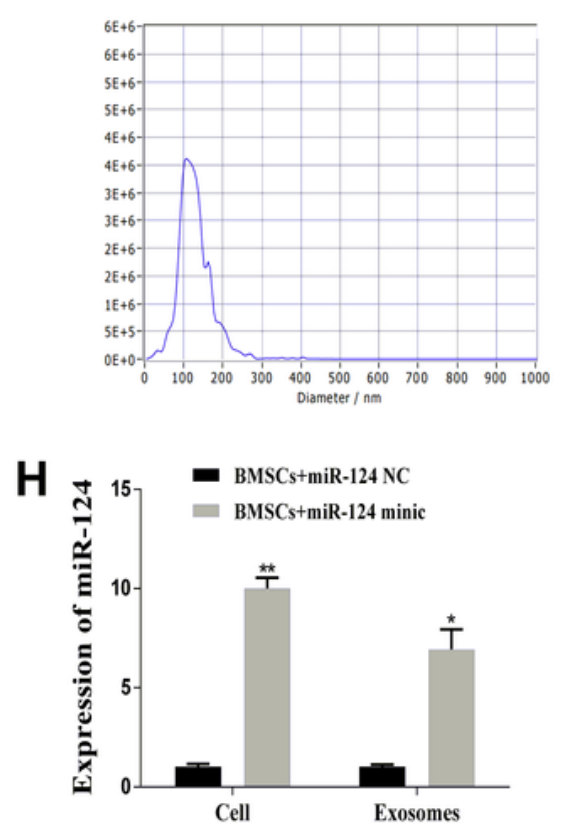

E

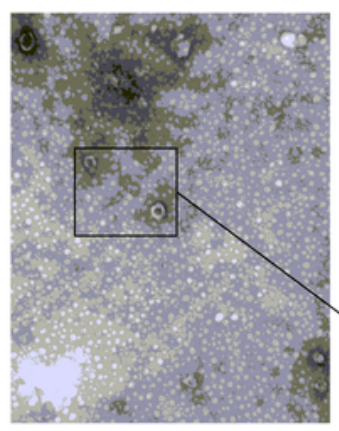

G

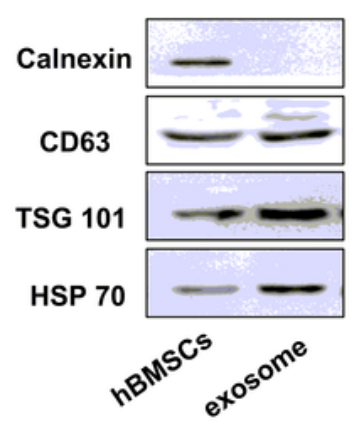

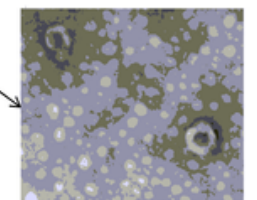

\section{Figure 6}

Isolation and identification of hBMSC-derived exosomes. a Antibodies against CD105, CD90, CD31, CD34, CD45, CD166, CD29, CD11b, HLA-DR, and CD73 were used to identify surface antigens by flow cytometry. 
CD29, CD90, CD105, CD166, and CD73 were positively expressed, while CD34, CD31, CD45, CD11b, and HLA-DR were negatively expressed. b The morphology of hBMSCs. A relatively large number of purified cells with a shuttle shape and swirling arrangement were observed. c-d The ability to induce adipogenic differentiation and osteogenic differentiation. A large number of lipid droplets appeared in the cells, and oil red $\mathrm{O}$ staining confirmed that the hBMSCs exhibited adipogenic differentiation (c). Many red calcium nodules were observed at the cell center using alizarin red staining (d). e Transmission electron microscopy (TEM) was used to identify BMSC-derived exosomes. The shape of the exosomes was globular or oval, with a complete lipid membrane. f Nanoparticle tracking analysis (NTA). The Zeta View nanoparticle tracking analyzer revealed that the exosome particles were predominately approximately $100 \mathrm{~nm}$ in size. g Western blot analysis. The exosome surface marker proteins CD63, TSG 101, and Hsp70 were expressed in BMSC-derived exosomes, whereas calnexin was not. $\mathrm{h}$ The miR-124-3p level in miR-124-3p-transfected hBMSCs and the exosomes derived from miR-124-3p mimic-treated hBMSCs was significantly higher than that in control cells. 
A

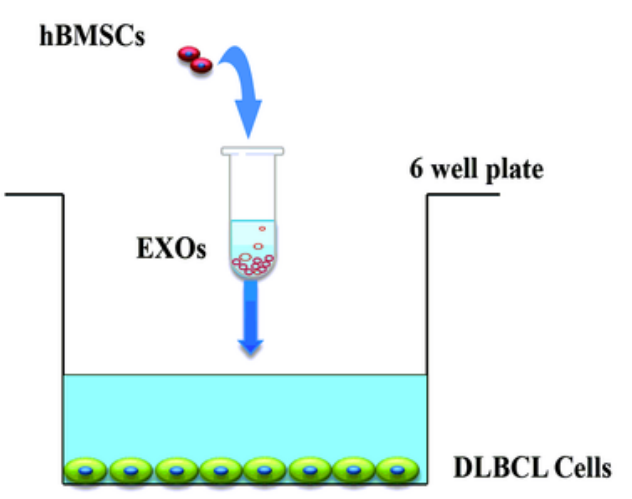

B
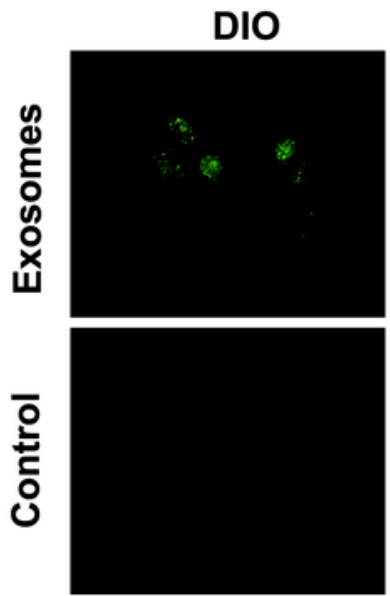

DAPI
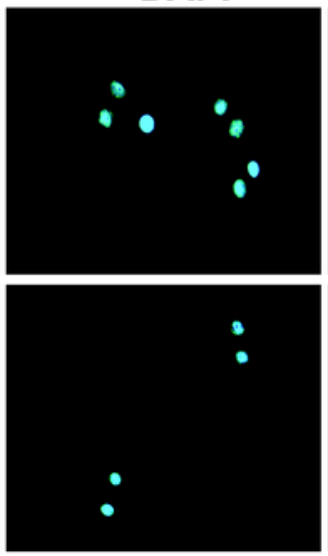

Merge
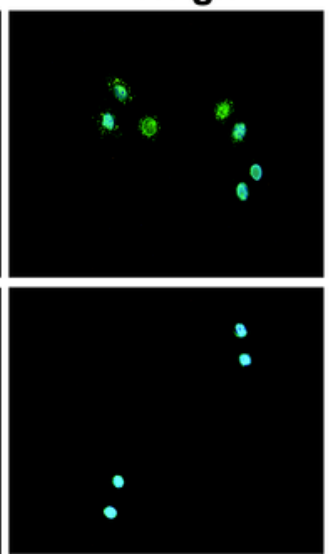

C
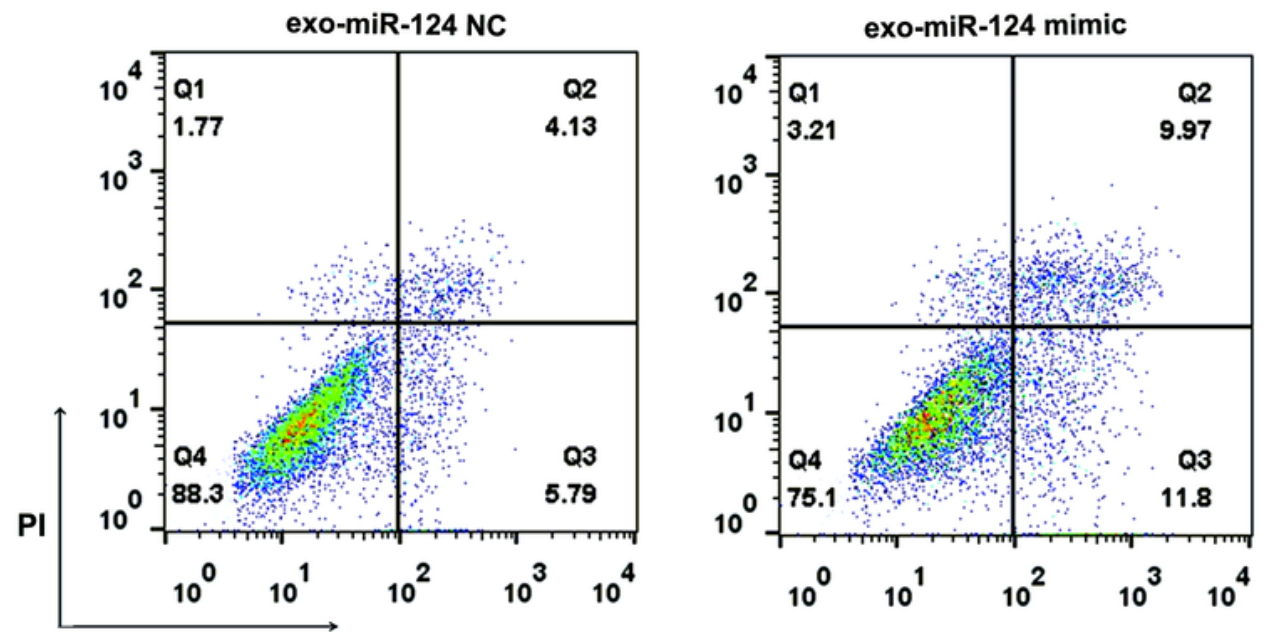

exo-miR-124 NC

exo-miR-124 mimic

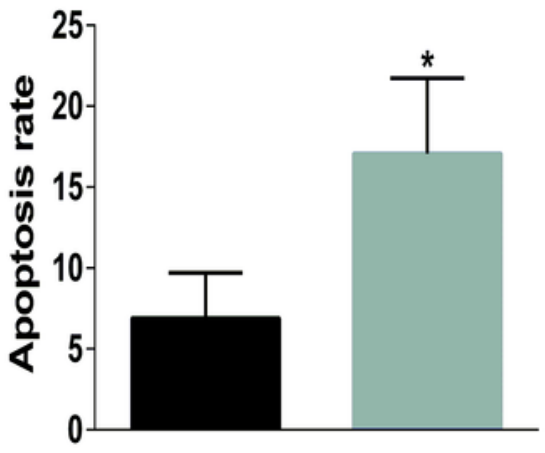

Annexin-V

D

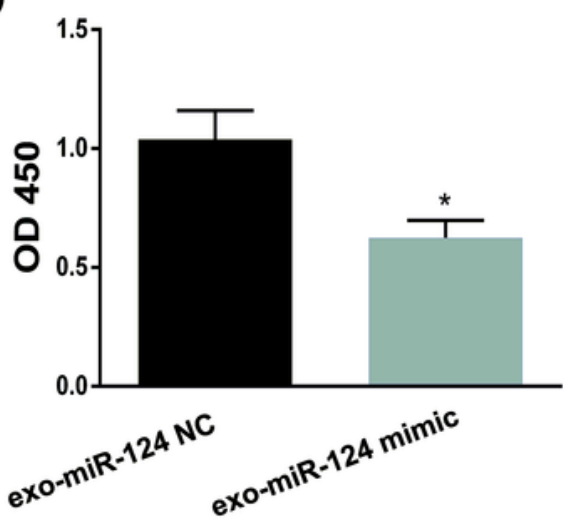

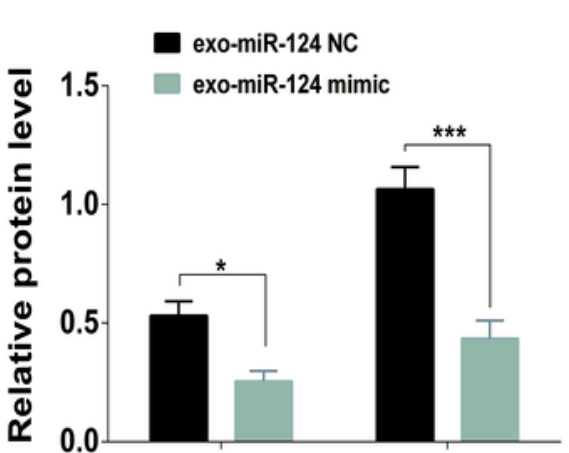

$e^{x-m i R-124 N C} e^{x-m i R-124 m i m i c}$

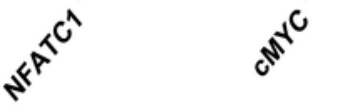

Figure 7

hBMSC-derived exosomal miR-124-3p suppresses DLBCL cell proliferation and enhances apoptosis. a Schematic diagram of the coculture process. $b$ Exosomes labeled with DIO absorbed by DLBCL cells under a fluorescence microscope $(\times 400)$. Slight green fluorescence, indicating exosomes absorbed by DLBCL cells, could be observed under a confocal fluorescence microscope. c-d Apoptosis and proliferation of DLBCL cells treated with hBMSC-derived exosomes containing miR-124-3p. The apoptosis 
rates were increased and the proliferation of DLBCL cells was inhibited after hBMSC-derived exosomes containing miR-124-3p were added. e Protein expression of NFATc1 and cMYC in DLBCL cells determined by western blot analysis. The protein expression levels of NFATc1 and CMYC were suppressed in DLBCL cells cocultured with hBMSC-derived exosomal miR-124-3p. Data are presented as the mean \pm SD of three independent experiments ( $\mathrm{P}<0.05$, ***P $<0.001)$.

A

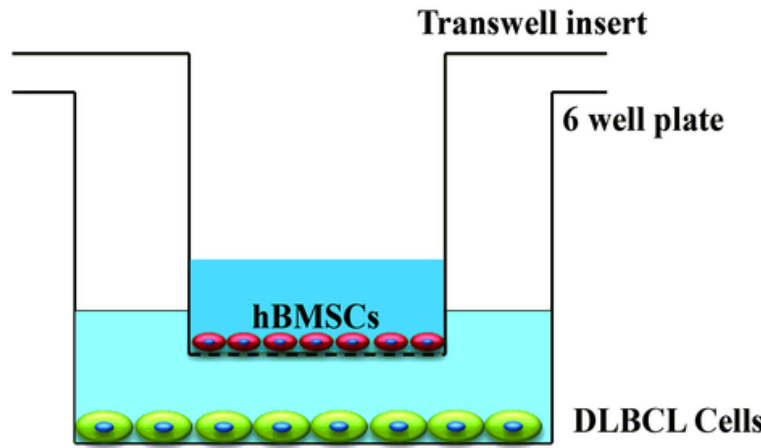

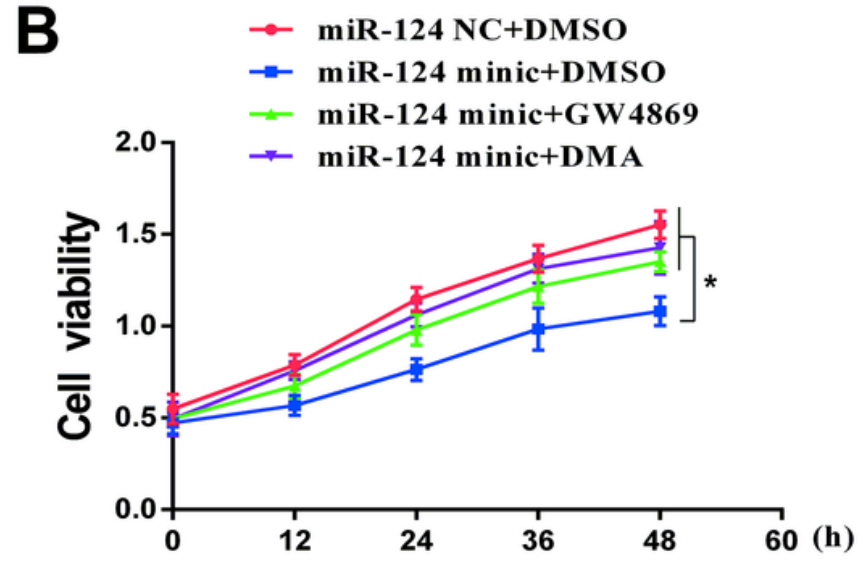

C

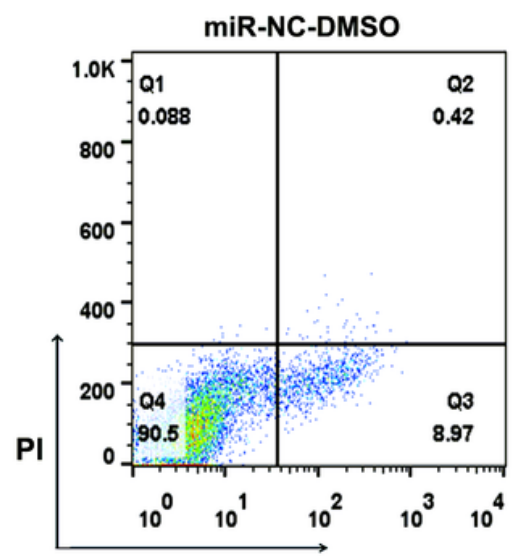

Annexin-V

D

$$
\begin{aligned}
& \text { miR-NC+DMSO } \\
& \text { miR-124 minic+DMSO } \\
& \text { miR-124 minic+GW4869 } \\
& \text { miR-124 minic+DMA }
\end{aligned}
$$

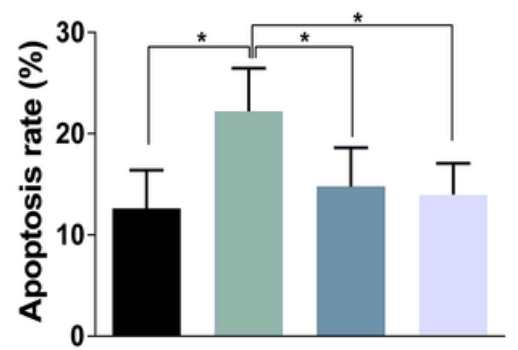

miR-124 mimic+DMSO

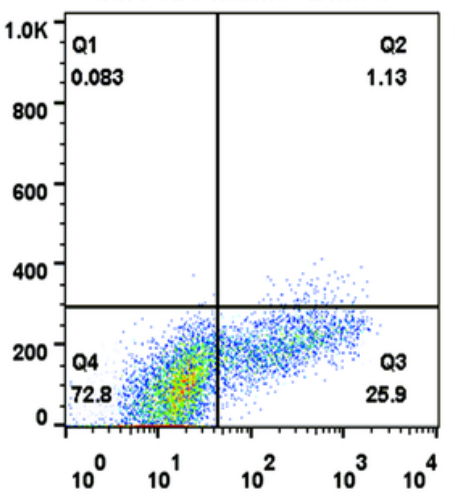

miR-124 mimic+GW4869

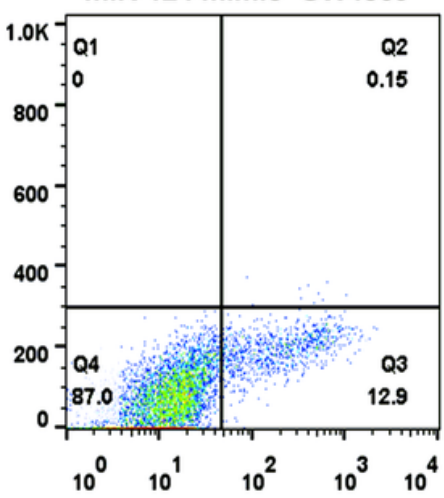

miR-124 mimic+DMA

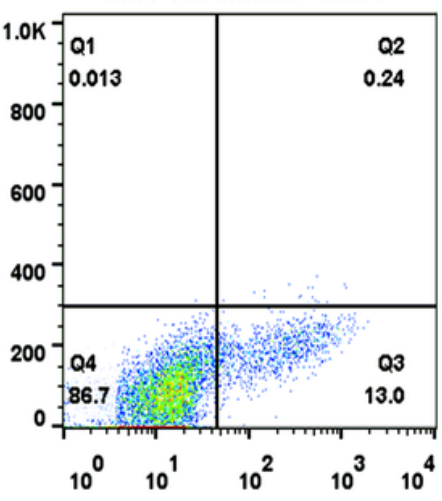

E

$$
\begin{array}{ll} 
& \text { miR-NC+DMSO } \\
& \text { miR-124 minic+DMSO } \\
& \text { miR-124 minic+GW4869 } \\
& \text { miR-124 minic+DMA }
\end{array}
$$

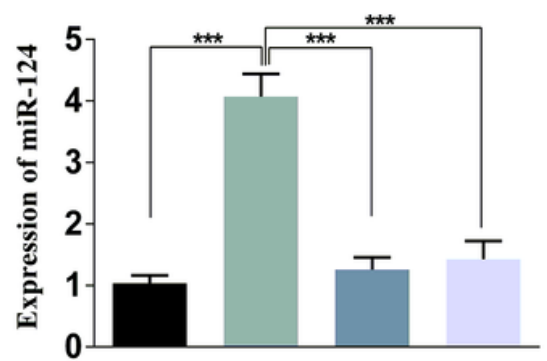

$\mathbf{F}$

$$
\begin{aligned}
& \text { miR-124 minic+DMSO } \\
& \text { miR-124 minic+GW4869 } \\
& \text { miR-124 minic+DMA }
\end{aligned}
$$$$
\text { - miR-124 NC+DMSO }
$$

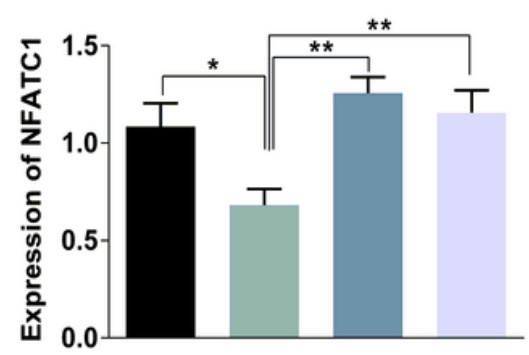

Figure 8

GW4869 and DMA suppress the production of exosomes from hBMSCs, thus reducing the delivered exosomal miR-124-3p and influencing their biological functions. a Schematic diagram of the coculture 
process. b-d The effects of hBMSC-derived exosomal miR-124-3p on cell proliferation and apoptosis could be rescued by GW4869 or DMA. e-f GW4869 and DMA treatment resulted in a significant decrease in miR-124-3p and increase in NFATc1. Data are presented as the mean \pm SD of three independent experiments $\left({ }^{*} P<0.05,{ }^{*} P \mathrm{P}<0.01\right.$, $\left.* \star * P<0.001\right)$.

A
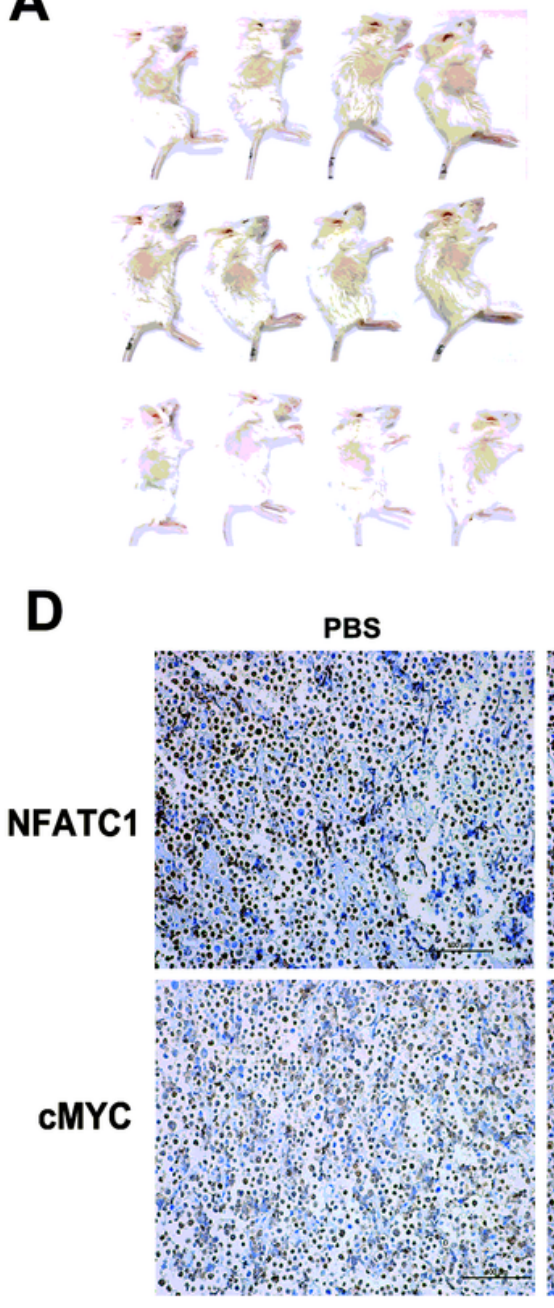

B

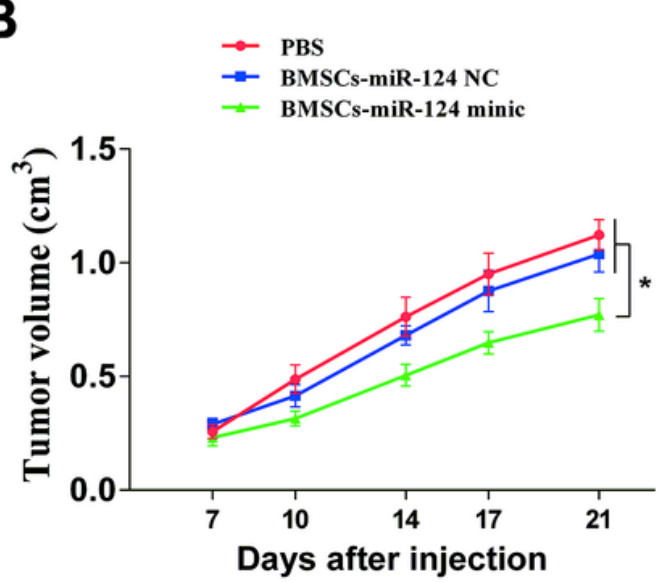

BMSCs+miR-124 NC
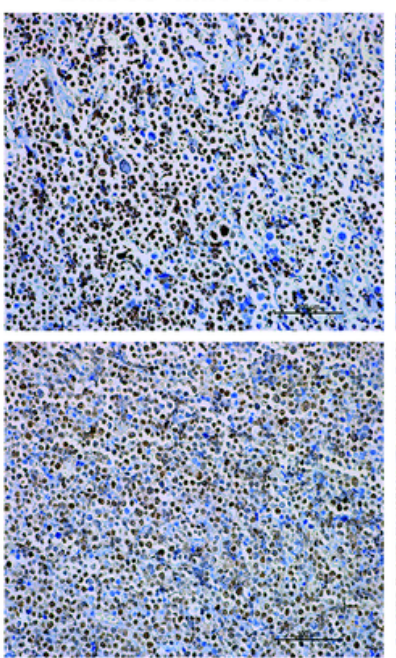

BMSCs+miR-124 mimic

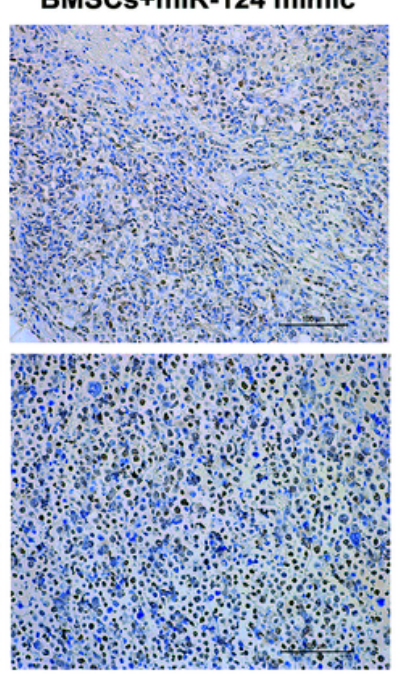

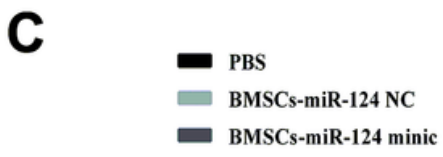
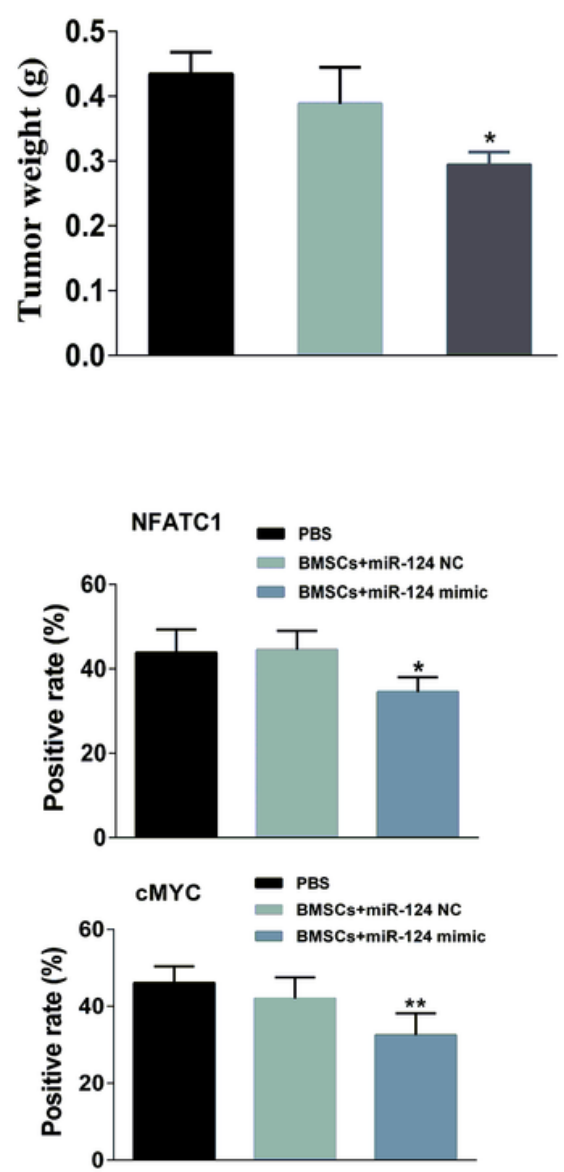

\section{Figure 9}

hBMSC-derived exosomal miR-124-3p delays tumor growth in vivo. a Representative images of xenograft tumors in different groups. b-c Tumor volumes and tumor weights in mice. In The tumor volumes and tumor weights in the hBMSC-derived miR-124-3p group were significantly lower than those in the hBMSCderived miR-124-3p NC and PBS groups. $d$ Expression of NFATc1 and cMYC in xenograft tumors determined by immunohistochemical staining $(\times 200)$. Data are presented as the mean \pm SD of three independent experiments $\left({ }^{*} P<0.05, * * P<0.01, * \star * P<0.001\right)$. 

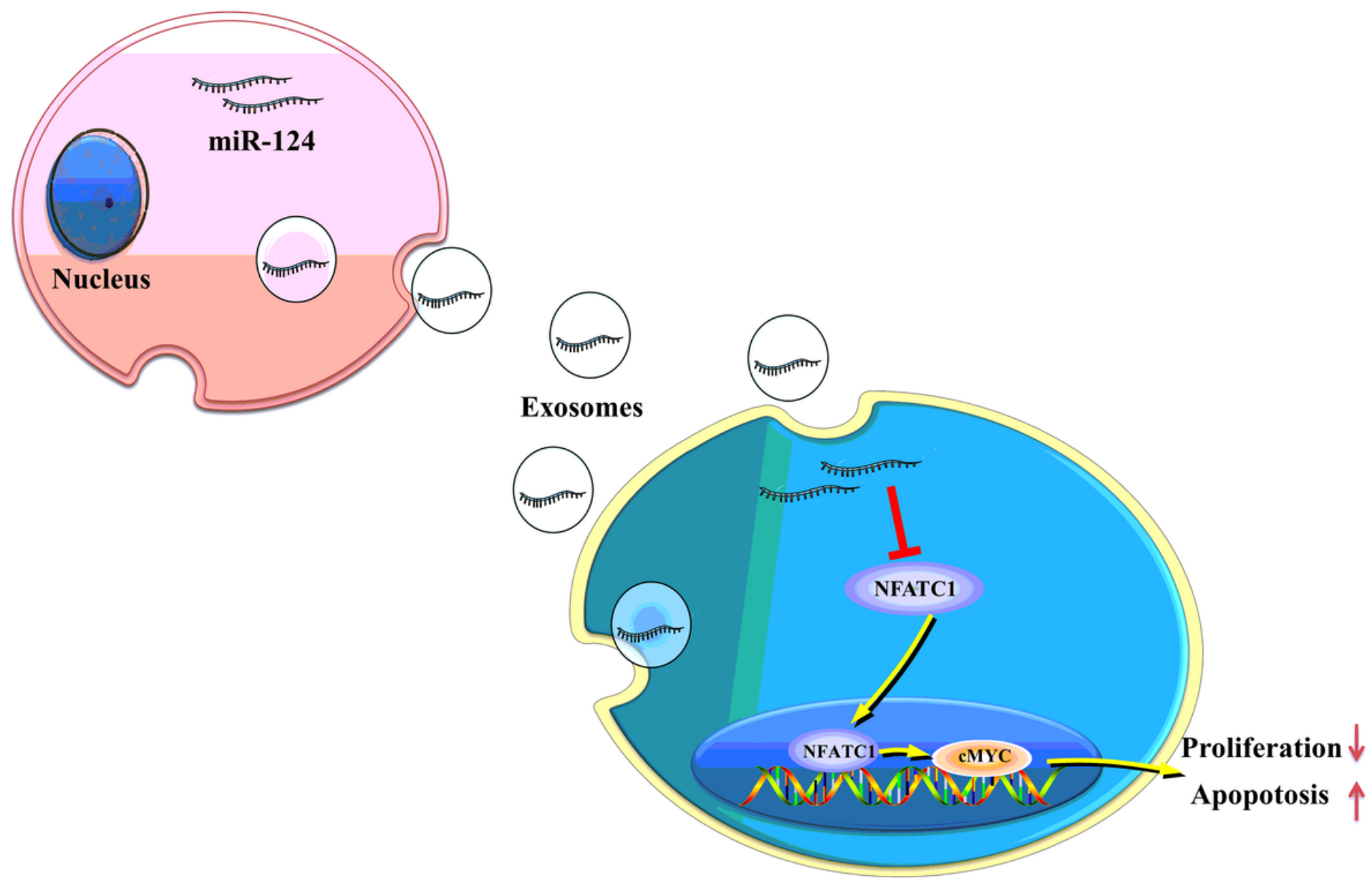

Figure 10

Diagram of the regulatory mechanism of hBMSC-derived exosomal miR-124-3p in DLBCL cells. hBMSCderived exosomal miR-124-3p decreases the expression of cMYC by downregulating NFATc1, thus inhibiting proliferation and enhancing apoptosis. 